\title{
Fachdidaktische Massnahmen im Französischunterricht aus Schülersicht: Der Zusammenhang mit Schulleistung und Motivation
}

\section{Alois Niggli, Rico Cathomas, Ulrich Trautwein und Inge Schnyder}

Autorenhinweis: Die vorliegende Studie wurde mit Mitteln des Schweizerischen Nationalfonds zur Förderung der wissenschaftlichen Forschung (Projekt Nr. 13DPD3114176/1) unterstützt.

Die jüngere Forschung zur Unterrichtsqualität im Fremdsprachenbereich weist auf die Notwendigkeit hin, die fachübergreifenden Qualitätsdimensionen des Unterrichts durch fachspezifische Beschreibungsmodelle zu ergänzen. In der vorliegenden Studie werden theoretische Annahmen über fachdidaktische Determinanten von Leistung und Interesse im Fremdsprachenunterricht anhand einer Stichprobe von 1 655 Achtklässlern aus den drei Schweizer Kantonen Freiburg, Luzern und Wallis überprü̈t. Auf faktorenanalytischem Wege konnten für das Fach Französisch als Fremdsprache folgende fünf Faktoren extrahiert werden: Gebrauch der französischen Sprache im Unterricht, Intensität des Übens und Anwendens, Einbezug lebensweltlicher Materialien, Vokabellernen und Kommunikation mit Fremdsprachigen. Mittels anschliessender Mehrebenenanalysen konnte gezeigt werden, dass der Gebrauch des Französischen und intensives Üben/Anwenden statistisch signifikant mit einer günstigen Entwicklung von Leistung und des Interesses im Verlaufe eines Jahres assoziiert waren. Ohne Einfluss blieb das Lernen von Vokabeln. Erwartungswidrig fand sich ein negativer Zusammenhang zwischen der Kommunikation mit Fremdsprachigen und der Entwicklung von Interesse an der betreffenden Sprache.

\section{Einleitung}

Die Förderung der Fähigkeit zur Kommunikation in einer fremden Sprache wird von einem breiten gesellschaftlichen Konsens getragen (Meyer, 2001) und den Schulen in zunehmendem Masse als zentrales Bildungsanliegen zugewiesen. Dementsprechend wird der Fremdsprachenunterricht mit einem hohen Einsatz an menschlichen und finanziellen Ressourcen an den Schulen implementiert (Finkbeiner, 2003; Grin, 1999). Wie erfolgreich die Schulen in der Vermittlung 
von Fremdsprachen sind, ist allerdings umstritten. Einerseits gibt es ernsthafte Zweifel an der Effizienz des gängigen schulischen Fremdsprachenunterrichts (Bleyhl, 2005; Meyer, 2001; Tschirner, 2004), andererseits wurde aber auch vor übertriebenen Erwartungen an die schulischen Möglichkeiten beim Erwerb einer zweiten Sprache gewarnt (Lightbown, 2000). In dieser Situation ist der Versuch, sich vermehrt Rechenschaft über das erreichte Leistungsniveau zu verschaffen (EDK, 2004), folgerichtig.

Für ein umfassenderes Verständnis der Wirksamkeit des gängigen Unterrichts muss neben die Überprüfung des Leistungsstands eine Analyse von leistungsförderlichen Merkmalen des Unterrichts treten. Es ist bemerkenswert, dass sich in der empirischen Unterrichtsforschung relativ wenige Studien zum Erwerb von Fremdsprachen finden lassen. Eine mögliche Erklärung dafür könnte in der Schwierigkeit liegen, Sprachkompetenz breit und überzeugend zu erfassen (Helmke \& Schrader, 2002). Mit dem Lernen von Fremdsprachen haben sich denn auch mehrheitlich Forschende beschäftigt, deren zentrale Referenzwissenschaften die Angewandte Linguistik, die Psycholinguistik oder die Kognitive Linguistik sind (Vollmer et al., 2001). Im Rahmen dieser disziplinären Zugänge befasst man sich primär mit den Grundlagen des Erwerbs einer Fremdsprache. Für leistungsdiagnostische Zwecke dürften jedoch auch sogenannte «Statusstudien» (Finkbeiner, 2003) bzw. Schulleistungsstudien erforderlich sein, mit denen eine gängige Unterrichtspraxis oder etablierte theoretische Vorstellungen, an denen sich Lehrkräfte orientieren, geprüft werden können. Im Rahmen dieser Studien lässt sich ermitteln, was Lehrkräfte konkret tun und welchen Erfolg sie mit ihren Massnahmen erzielen.

Die psychologisch orientierte Lehr-Lernforschung stützt sich bei der Erforschung von Determinanten des Lernerfolgs bislang vielfach auf Daten im Fach Mathematik (Helmke \& Schrader, 2002). Es stellt sich die Frage, inwieweit anhand der Mathematik gewonnene Befunde auch für andere Fächer Geltung beanspruchen können. Kunter und Baumert (2006a) argumentieren, dass eine Klassifikation unterrichtsrelevanter Merkmale am vielversprechendsten durch eine Kombination von generellen Indikatoren mit domänenspezifischen Befunden erreicht werden könne. Neben bekannten allgemeinen Unterrichtsfaktoren (Brophy \& Good, 1986; Helmke \& Schrader, 2001; Helmke \& Weinert, 1997) dürften jeweils auch domänenspezifische Adaptionen oder Ergänzungen erforderlich sein. Der vorliegende Beitrag stellt eine Konzeptualisierung fachspezifischer Determinanten von Schulleistung und Interesse im Fremdsprachenunterricht vor und prüft diese empirisch anhand einer grossen Schulleistungsstudie. 


\section{Allgemeine und fachspezifische Determinanten schulischer Leistung und schulischen Interesses}

Die Determinanten schulischer Leistungen lassen sich in eher distale Faktoren (z.B. Schulform bzw. -niveaus) und proximale Faktoren (z.B. Unterrichtsqualität) unterteilen. Schulleistungsstudien der jüngeren Zeit haben eine Reihe von distalen Determinanten für Leistungsfortschritte im Fremdsprachenunterricht identifiziert (Köller, Baumert, Cortina, Trautwein \& Watermann, 2004; Köller \& Trautwein, 2004; Köller, Trautwein, Cortina \& Baumert, 2006). Auf die Rolle von unterschiedlichen Lernopportunitäten haben Trautwein, Köller, Lehmann und Lüdtke (2007) hingewiesen. Ihre Studie zeigte Unterschiede zwischen Abiturienten in Grund- und Leistungskursen sowie zwischen Absolventen unterschiedlicher Schulformen auf. Neumann et al. (2007) konnten die Bedeutung institutioneller und kompositioneller Bedingungen für differenzielle Entwikklungsverläufe im Fach Französisch als Fremdsprache aufzeigen. Kompositionseffekte entstehen durch Unterschiede in der Zusammensetzung einer Schulklasse. Effekte der Institution sind durch Unterschiede zwischen verschiedenen Bildungsgängen bedingt. Massgebend dürften unterschiedliche Anforderungen in den Curricula, Unterschiede in der Lehrerausbildung sowie damit korrespondierende Unterrichtskulturen sein (Neumann \& Trautwein, 2007). Eine Besonderheit des Fremdsprachenlernens liegt darin, dass es auch über ausserschulische Erfahrungen stattfinden kann (Köller \& Trautwein, 2004). So belegen Befunde zum Auslandsaufenthalt von Oberstufenschülern die grosse Bedeutung des Lernens in authentischen Kontexten (Köller et al., 2004). Spitzenleistungen im Fach Englisch wurden auch in der Studie von Trautwein et al. (2007) nur von Abiturienten erbracht, die einen nennenswerten Auslandsaufenthalt absolviert hatten.

Studien zu proximalen Einflüssen, die auch das gängige Unterrichtsgeschehen erfassen (Schnabel, 2001), können die Wirkmechanismen zusätzlich erhellen. In der Studie Deutsch-Englisch-Schulleistungen-International (DESI; Klieme et al., 2006) wurden Informationen über Unterrichtsmerkmale sowohl mit Hilfe von Fragebögen als auch mit Videobeobachtungen gewonnen. Zielkriterium im Englischunterricht war grösstenteils das «Hörverständnis». Die Bedeutung sogenannter Basisdimensionen der Unterrichtsqualität, die in jedem Fach zum Lernerfolg beitragen sollten, wurde grundsätzlich bestätigt. Es handelt sich dabei unter anderem um die Störungsfreiheit, hohe Aufgabenorientierung und ein positives Fehlerklima. Zusätzlich kennzeichnend für den Fremdsprachenunterricht ist aber auch der Gebrauch der Zielsprache. Ihre Vernachlässigung, also die häufige Verwendung der deutschen Sprache im Englischunterricht, stand in einem negativen Zusammenhang mit dem Hörverständnis. In bestimmten funktionalen Kontexten (Erklärungen bei Aufgaben, Übergang zu einem neuen Thema) zeigten sich allerdings auch positive Effekte. Deutlich variierten auch die Sprechanteile zwischen Lehrpersonen, Schülerinnen und Schü- 
lern. Lehrpersonen sprachen mehr als doppelt so lange wie ihre Schülerinnen und Schüler. 84 Prozent ihres Sprechanteils erfolgte auf Englisch, während bei den Schülerinnen und Schülern dieser Anteil bei etwa 75 Prozent lag. Tendenziell unterschätzten die Lehrpersonen ihre Sprechanteile. Positiv auf die Entwikklung der Hörverstehensleistungen wirkten sich auch Lehrer-Schüler-Dialoge aus, die über einfache Frage-Antwort-Sequenzen hinausgehen.

Darüber hinaus gibt die DESI-Studie auch Anlass, über die Fachspezifität von Qualitätsfaktoren nachzudenken. So kam dem Ausmass der Strukturierung im Englischunterricht - anders als Befunde zum Fach Mathematik nahelegen keine positive Rolle zu. Ebenso wirkte sich die Häufigkeit der Gruppenarbeit weder auf die Entwicklung der Sprachkompetenzen noch auf die Verbesserung des Lerninteresses aus. Dass Gruppenarbeit unter Umständen sogar nachteilig sein kann, bestätigte auch eine Arbeit von Ellis (2005). Eng geführte Fragen waren in der DESI-Studie dagegen positiv mit den Englischleistungen assoziiert. Auch dieses Ergebnis widerspricht den Befunden der empirischen Unterrichtsforschung im Fach Mathematik.

Hinweise auf fachspezifische Qualitätsdimensionen fanden sich auch bei Campbell, Kyriakides, Muijs und Robinson (2005). Diese Autoren analysierten den Einfluss des Lehrerverhaltens im Fach Mathematik und im Unterricht der Erstsprache. Informative Mitteilungen, das Frageverhalten, die Vergabe von Feedback, die Bereitstellung von Anwendungsmöglichkeiten sowie die Organisation der Lektionen hatten in sprachlichen Fachkontexten eine wesentlich geringere Bedeutung als im Fach Mathematik.

Die differenziellen Befunde für die Mathematik und den Fremdsprachenunterricht machen deutlich, wie wichtig die Berücksichtigung einer fachspezifischen Perspektive in der Unterrichtsforschung ist. Eine Reihe von Befunden zum Fremdsprachenlernen stammt aus der linguistischen Forschung. Eine zentrale Gemeinsamkeit daraus abgeleiteter Empfehlungen ist, dass der wirksame Fremdsprachenunterricht durch eine hohe Lernintensität sowie durch kommunikativ-inhaltsorientierte Anforderungen gekennzeichnet ist (Judd, Tan \& Walberg, 2001). Norris und Ortega (2001) präzisierten diese Aussagen durch eine umfangreiche Metaanalyse, mit der sie zwei zentrale theoretische Kontroversen über die Praxis des Zweitsprachenerwerbs zu klären versucht haben, nämlich, ob der Erwerb einer zweiten Sprache eher von direkt-explizit (mit deduktiven und induktiven Elementen) oder von indirekt-implizit gesteuerten Lernvorgängen abhängt und welche der beiden Fokusstrategien, Focus-on-Forms (FonFs) oder Focus-on-Form (FonF), dabei effektiver ist. Bei den FonFs-Strategien handelt es sich um metasprachliche Erläuterungen der Form und um Übungen anhand nicht kontextualisierter Beispiele (beispielsweise grammatikalische Formen in inhaltlich unverbundene Sätze einsetzen). Mit sogenannten FonF-Methoden werden Formen in ihrer Kontextualisierung wahrgenommen und gelernt. So können die Schülerinnen und Schüler zum Beispiel veranlasst werden, einen zusammenhängenden Text von der Gegenwart in die Zukunft zu transformieren. 
Die damit konstruierbaren vier Strategien konnten in die folgende Effektreihe überführt werden: Explizit FonF > explizit FonFs > implizit FonF > implizit FonFs. Norris und Ortega gaben allerdings zu bedenken, dass die in den verwendeten Messinstrumenten verlangten Leistungen möglicherweise explizite Treatments favorisierten (Norris \& Ortega, 2000). Auch wurden in lediglich 16 Prozent der Studien Angaben zur Reliabilität der abhängigen Leistungsvariable gemacht (Norris \& Ortega, 2001).

Neben der Entwicklung fachlicher und kognitiver Kompetenzen bemisst sich die Wirksamkeit und Qualität des Unterrichts auch nach affektiven, sozialen und motivationalen Zielkriterien (Finkbeiner, 2001; Klieme et al., 2006). Aus dem breiten Spektrum der Möglichkeiten wird deshalb in diesem Beitrag auch das Interesse am Fach in die Analysen mit einbezogen. Nach übereinstimmenden Befunden verschiedener Arbeiten erweist sich das Interesse im Zusammenspiel mit kognitiven Aspekten als bedeutsamer Faktor (Eccles \& Wigfield, 2002; Köller, Trautwein, Lüdtke \& Baumert, 2006). Schiefele, Krapp und Schreyer (1993) fanden in ihrer Metaanalyse in den Fremdsprachenfächern eine durchschnittliche Korrelation von .33 zwischen Leistung und Interesse. Dieser Zusammenhang gewinnt in der Sekundarstufe I zunehmend an Bedeutung (Marsh, Trautwein, Lüdtke, Köller \& Baumert, 2005). Im Gegensatz zur Leistung scheint Interesse durch allgemeines Instruktionsverhalten wie beispielsweise das Klassenmanagement jedoch wenig beeinflusst zu werden (Kunter \& Baumert, 2006b). Domänenspezifische Komponenten gewinnen daher auch für die Interesseförderung an Bedeutung. Zu nennen sind vor allem als sinnvoll und authentisch erlebte oder kognitiv angemessen anspruchsvolle Aufgabenstellungen, die persönliches Beteiligtsein ermöglichen (Blömeke, Risse, Müller, Eichler \& Schulz, 2006; Hidi \& Renninger, 2006; Tsai, Kunter, Lüdtke, Trautwein \& Ryan, in press). Als besondere Spezifität des Fremdsprachenunterrichts werden in diesem Zusammenhang auch Übungen zur interkulturellen Kommunikation wie beispielsweise Klassenkorrespondenzen empfohlen (Grau \& Würffel, 2003).

\section{Fachdidaktische Determinanten der Lern- und Interessenförderung: Eine theoretische Rahmen- konzeption}

Im Folgenden entwerfen wir eine theoretische Rahmenkonzeption, die spezifisch für didaktische Besonderheiten des Fremdsprachenunterrichts gilt und die wir anschliessend empirisch prüfen. Sie stützt sich auf drei fachdidaktisch bedeutsame Merkmale der fremdsprachlichen schulischen Lernumwelt: (1) Die Intensität der Interaktion in der Zielsprache, (2) die Qualität der sprachlichen Anforderungen im Rahmen von sogenannten Sprachfeldern sowie (3) den zusätzlichen Einbezug authentischer medialer Erfahrungsmöglichkeiten. 
(1) Intensität der Interaktion: Seit den 70er Jahren, bzw. seit der sogenannten kommunikativen Wende, wird unter dem Begriff «Kommunikative Kompetenz» ein übergeordnetes Lernziel des Lernens einer Fremdsprache verstanden (Piepho, 1976). Diese pragmatische Orientierung macht sprachliches miteinander Handeln zum Kernstück des Unterrichts. Dabei wird der Akzent einerseits auf die Interaktion der Lernenden gesetzt (Schiffler, 1985), aber auch der Lehrperson kommt als sprachliches Modell grosse Bedeutung zu (Lightbown, 2000). Die Intensität des kommunikativen Gebrauchs der Fremdsprache bildet sozusagen die Hintergrundfolie des Sprachunterrichts (siehe Ellis, 2005; Judd et al., 2001; Ziberi-Luginbühl, 1999). Allerdings wird damit noch nicht beantwortet, in welcher Qualität der Umgang mit Sprache stattfindet und welche Fertigkeiten gefordert sind (Levelt, 1989).

(2) Qualität der sprachlichen Anforderungen: Vor allem aufgrund der erwähnten Befunde der DESI-Studie gilt es in erster Linie, gängige Annahmen der Unterrichtsforschung zur kognitiven Aktivierung fachspezifisch zu konzeptualisieren. Für die Beherrschung von Fremdsprachen bietet sich dazu die Taxonomie von Valette und Disick (1972) an. Ihr Ansatz unterscheidet folgende Stufen der kognitiven Ich-Beteiligung: (1) Mechanical Skills (passives Wahrnehmen, reproduzieren); (2) Knowledge (Wiedererkennen, automatisches Abrufen); (3) Transfer (situativ angemessen abrufen); (4) Communication (eigene Gedanken ausdrücken); (5) Criticism (über Sprache nachdenken, abstrakte Diskurse führen). Diese kognitiven Stufenbereiche lassen sich mit den beiden sprachwissenschaftlichen Varianten des kontexteingebetteten bzw. dekontextualisierten Sprachgebrauchs in der Schule kombinieren (Cummins, 2001). Mit Hilfe dieser beiden Dimensionen werden in der folgenden Abbildung 1 vier Felder konstruiert, die fachdidaktisches Handeln im Unterricht qualitativ beschreiben.

\begin{tabular}{|c|c|c|}
\hline $\begin{array}{l}\text { Feld I: Einfache } \\
\text { Anforderungen mit } \\
\text { Situationsbezug } \\
\text { (BICS) }\end{array}$ & 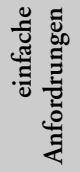 & $\begin{array}{l}\text { Feld II: Einfache, } \\
\text { kontextreduzierte } \\
\text { Anforderungen; } \\
\text { Automatisierung }\end{array}$ \\
\hline $\begin{array}{l}\text { Kontexteingebettet } \\
\text { (konkret) }\end{array}$ & & $\begin{array}{l}\text { Dekontextualisiert } \\
\text { (abstrakt) }\end{array}$ \\
\hline $\begin{array}{l}\text { Feld III } \\
\text { Komplexere Transfer- } \\
\text { Anforderungen mit } \\
\text { Situationsbezug }\end{array}$ & 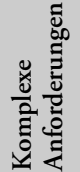 & $\begin{array}{l}\text { Feld IV } \\
\text { Sprachliche Bewälti- } \\
\text { gung komplexer } \\
\text { Fragestellungen } \\
\text { (CALP) }\end{array}$ \\
\hline
\end{tabular}

Abbildung 1: Felder der Sprachlernaktivität nach den Dimensionen kognitiver Ich-Beteiligung und zunehmender Dekontextualiserung 
Das sogenannte BICS-Feld I (basic interpersonal communicative skills) beinhaltet eher einfache lebensweltliche Routinen (Cummins; 1979, 2001). Dies gilt auch für das Lernen sprachsystematischer Formen nach der "Focus on Form»-Strategie, zum Beispiel wenn eine sprachliche Form in einen typischen alltäglichen Kontext eingebettet ist. Das Automatisierungsfeld II dagegen umfasst im Wesentlichen dekontextualisierte "Focus on FormS»-Übungen in Grammatik sowie Wortschatztrainings (Haudeck, 2005). Im Kommunikationsfeld III werden die kognitiven Anforderungen hingegen differenzierter. $\mathrm{Zu}$ meistern sind komplexere Alltagssituationen. Im Rahmen einer Austauschdidaktik können in diesem Feld auch Kontakte mit Menschen, die die Fremdsprache sprechen, arrangiert werden. Im CALP-Feld IV (cognitive academic language proficiency) schliesslich sind nach Cummins (1979) anspruchsvolle Leistungen durch den Rückgriff auf rein formale sprachliche Rationalitätsansprüche zu erbringen.

(3) Authentische Erfahrungsmöglichkeiten: Die Felder I und III lassen sich durch die Berücksichtigung authentischer Medien zusätzlich mit Elementen aus der Lebenswelt der Lernenden anreichern (Edmondson \& House, 2000; Stern, Eriksson, Le Pape Racine, Reutener \& Serra, 1999). Diese Sprachprodukte eröffnen zugleich Anschlussmöglichkeiten für den interkulturellen Austausch im ausserschulischen Alltag (Lüdi, Pekarek \& Saudan, 2001).

\section{Ableitung der Fragestellung}

Datenbasis für die nachfolgenden Fragestellungen ist eine Studie zur Wirkung von Hausaufgaben, in der bei Achtklässlern zu zwei Messzeitpunkten Französischtests zum Einsatz kamen (Schnyder, Niggli, Cathomas, Trautwein \& Lüdtke, 2006; Schnyder, Niggli, Cathomas \& Trautwein, 2006). Zudem wurde über Fragebogeninstrumente die Entwicklung der schulischen Motivation der Schülerinnen und Schüler erfasst. Auch die Erfassung der fachdidaktischen Elemente des Französischunterrichts erfolgte über den Einsatz von Schülerfragebögen. Effekte fachdidaktischer Massnahmen stehen im Mittelpunkt der vorliegenden Arbeit. Aufgrund der gewählten Zielstichprobe der Volksschule wurde im Hinblick auf die Konzeption der Sprachfelder entschieden, CALP-Anforderungen unberükksichtigt zu lassen. Ein dekontextualisierter Umgang auf diesen anspruchsvollen kognitiven Stufen wird erst nach rund 5000 Unterrichtsstunden erreicht (Zydatiss, 2000). Dies ist bis zum Ende der Sekundarstufe I in der Regel nicht der Fall. Die vier qualitativen Sprachfelder wurden somit auf drei reduziert.

Konkret verfolgten wir folgende Fragestellungen. Erstens überprüften wir die psychometrische Qualität des von uns entwickelten Fragebogens zu fachdidaktischen Merkmalen des Französischunterrichts. Grundlage dazu bildet die postulierte theoretische Rahmenkonzeption. Zweitens untersuchten wir den $\mathrm{Zu}$ sammenhang zwischen den fachdidaktischen Dimensionen und der Hausaufgabenqualität als weiteres wichtiges Merkmal von Unterrichtsqualität. Da wir 
eine fachdidaktische Konzeption vertreten, die sich im Wesentlichen als Dimensionierung qualitativ unterschiedlicher Aufgabenstellungen versteht, sind Wechselbeziehungen mit der Praxis der Hausaufgabenvergabe evident. Zu der Hausaufgabenpraxis liegen im verwendeten Datensatz sowohl Schülerurteile als auch Expertenurteile vor. Wir gingen von der Annahme aus, dass die fachdidaktischen Skalen in einem mittleren Ausmass mit der schülerperzipierten Hausaufgabenqualität korrelieren sollten. Etwas niedrigere Korrelationen erwarteten wir mit den Expertenurteilen, da in diesem Fall eine Einschätzung nach methodischdidaktischen Kriterien der Profession vorgenommen wurde.

Drittens erwarteten wir einen Zusammenhang zwischen der Ausprägung der fachdidaktischen Skalen und der Entwicklung von Interesse und Leistung der Schülerinnen und Schüler. Dabei sollten sich der Gebrauch des Französischen sowie vielfältige kontextualisierte Übungs- und Anwendungsmöglichkeiten (Feld I und III) im Unterricht positiv auf die Fachleistungen und auch auf das Interesse auswirken. Dekontextualisiertes Üben (Feld II) sollte dagegen weder mit Leistung noch mit Interesse korrelieren. Schliesslich gingen wir von der $\mathrm{Hy}-$ pothese aus, dass sich der Einbezug authentischer Medien sowie authentische Kommunikationsmöglichkeiten im Rahmen des üblichen Unterrichtes (Feld III) positiv auf das Interesse auswirken dürften.

\section{Methode}

\section{Stichprobe}

Der verwendete Datensatz entstammt einer grösseren Studie zur Wirkung von Hausaufgaben im Fach Französisch als Fremdsprache, die von der Pädagogischen Hochschule Freiburg sowie der Abteilung Lehrerinnen- und Lehrerausbildung für die Sekundarstufe I an der Universität Freiburg in Kooperation mit dem Max-Planck-Institut für Bildungsforschung in Berlin durchgeführt worden ist. Insgesamt gingen in die vorliegenden Auswertungen die Daten von 1655 Achtklässlern (51,2 \% weiblich) aus 97 Schulklassen und 27 Schulen in den drei Kantonen Freiburg, Wallis und Luzern in die Auswertung ein. In den beiden Kantonen Freiburg und Wallis nahmen jeweils alle deutschsprachigen Schulen teil. Die Erhebung fand hier ausschliesslich in Klassen mit der Unterrichtssprache Deutsch statt. Im Kanton Luzern musste sich die Erhebung aus organisatorischen Gründen auf vier Schulen beschränken. Die Erhebungen der Französischleistungen und des Fachinteresses erfolgten im September 2003 (Erhebungszeitpunkt t1) und im Juni 2004 (Erhebungszeitpunkt t2). Der Fachdidaktikfragebogen wurde zu t2 eingesetzt. Die prädiktiven Befunde (HLMAnalysen) stammen von einem Subsample aus Schülerinnen und Schülern, die am Leistungstest zu t 1 und t 2 teilgenommen hatten und von deren Klassen Angaben zu den fachdidaktischen Dimensionen vorlagen. Insgesamt waren dies 1419 Schülerinnen und Schüler aus 92 Klassen. 


\section{Instrumente}

Fachdidaktikskalen. In der Unterrichtsforschung haben sich verschiedene Erfassungsperspektiven etabliert (Clausen, 2002). Unter anderem haben sich die Befragung der Schülerinnen und Schüler und die Aggregation dieser Urteile auf Klassenebene bewährt (Gruehn, 2000). Diese Strategie implementierten wir auch in der vorliegenden Studie. Von einem Fachteam (Forschungsverantwortliche, Dozierende der Fachdidaktik, Lehrkräfte, die Fremdsprachen unterrichten) wurde ein Pool von 30 Items zusammengestellt. Wegweisend war das oben beschriebene fachdidaktische Rahmenkonzept, das den Gebrauch der Unterrichtssprache Französisch, Anforderungen in den drei Sprachfeldern sowie die Berükksichtigung authentischer Medien umfasste. Zwei Items zum Gebrauch des Deutschen im fremdsprachlichen Unterricht wurden in Anlehnung an Edelstein (1970) formuliert. Aus derselben Studie wurde auch ein Item zum Briefeschreiben im Alltag übernommen. Bei den übrigen Items handelt es sich um Eigenentwicklungen. Die Items waren auf einer fünfstufigen Skala zu beantworten: «stimmt nicht», «stimmt ein wenig», «stimmt zur Hälfte», «stimmt ziemlich», «stimmt genau».

Qualität der Hausaufgaben. Die Schülerinnen und Schüler schätzten die Qualität der Hausaufgaben im Fach Französisch anhand von acht Items ein (Beispiel: «Die Hausaufgaben sind fast immer sehr gut ausgewählt.»). Die Erhebung erfolgte zum Messzeitpunkt t2. Verwendet wurde ein vierstufiges Antwortformat: «stimmt überhaupt nicht», «stimmt eher nicht», «stimmt eher», «stimmt ganz genau». Cronbach's Alpha betrug .84. Anhand von Vergabeprotokollen der Lehrkräfte wurde die Qualität der Hausaufgaben zusätzlich durch externe Rater beurteilt. Pro Lehrkraft wurden durchschnittlich 16 Aufgaben bewertet (Min. = 3, Max. = 37), die während des Schuljahres zu drei Erhebungszeitpunkten gesammelt worden waren. Jede Aufgabe wurde von zwei trainierten Ratern eingeschätzt. Verwendet wurden drei hoch inferente 5-stufige Ratingskalen: Kognitives Anspruchsniveau, Einbettung in den Lernprozess, Kontextbezug. Die Interraterkorrelationen betrugen .80 für das Anspruchsniveau, . 70 für die Einbettung in den Lernprozess und .84 für den Bezug zum Lebenskontext.

Interesse. Insgesamt zwölf Items wurden zur Erfassung des Interesses an Französisch eingesetzt (Beispielitems: «Ich möchte später einige Zeit in Frankreich verbringen.», «Ich meine, dass man den Stoff in Französisch auch später gut gebrauchen kann.», «Ich beschäftige mich sehr gerne mit Französisch.»). Die Formulierung der fachbezogenen Items erfolgte unter Bezug auf Krapp (1998) sowie Eccles und Wigfield (2002). Das Antwortformat entsprach demjenigen der Hausaufgabenqualitätsskala. Cronbach's Alpha zu t 1 und t 2 betrug .90 und .88 .

Leistungstest. Sowohl zu Beginn als auch am Ende des Schuljahres kam ein standardisierter curriculumbezogener Leistungstest zur Anwendung. Der Test bestand aus insgesamt $62(\mathrm{t} 1)$ und $48(\mathrm{t} 2)$ Aufgaben im Multiple-Choice- bzw. offenen Format. Die interne Konsistenz war zu beiden Messzeitpunkten sehr 
hoch (> .90). Die Skalierung der Tests erfolgte auf der Basis des einparametrischen Rasch-Modells (Neumann et al., 2007).

Kursniveau. Im Kanton Wallis werden bis Ende der achten Klasse zwei Leistungsniveaus geführt, während es in Freiburg und Luzern drei sind. In den Analysen wurde zwischen Grundansprüchen (Realschule bzw. Niveau II) und erweiterten Ansprüchen (Sekundarschule/Progymnasium bzw. Niveau I) unterschieden.

\section{Statistische Analysen}

Mit der Untersuchung der fachdidaktischen Massnahmen der Lehrkräfte zielt unsere Arbeit auf die Erfassung eines Unterrichtsmerkmals ab; es geht darum, Unterschiede in den Ausprägungen der fachdidaktischen Massnahmen zwischen den verschiedenen Klassen zu ermitteln und in Bezug zu weiteren Variablen zu setzen. Die fachdidaktischen Massnahmen sind also ein Klassenebene-Konstrukt (Cronbach, 1976), das über die Einschätzung der individuellen Schülerinnen und Schüler erfasst wurde. Für die weitergehenden Analysen mussten die Einschätzungen der Schülerinnen und Schüler deshalb auf Klassenebene aggregiert werden (Lüdtke, Robitzsch, Trautwein \& Kunter, 2007). Diese Aggregation erwies sich als gerechtfertigt, da vorgängige Reliabilitätsberechnungen zeigten, dass die Schülerwahrnehmungen eine ausreichend hohe Reliabilität, gemessen über die ICC(1) und ICC(2), aufwiesen (Lüdtke, Trautwein, Kunter und Baumert, 2006). Die Intraklassenkorrelation ICC(1) ist ein Mass für die Reliabilität eines einzelnen Schülerurteils. Es wird bestimmt, mit welcher Genauigkeit im Durchschnitt ein Schüler das entsprechende Unterrichtsmerkmal beurteilt. Ihr Medianwert lag bei 0.18 (Min. = 0.08; Max. =0.50). Die Intraklassenkorrelation ICC(2) gibt dagegen die Reliabilität des Klassenmittelwertes der Schülerurteile an. Hier lag der Medianwert bei 0.79 (Min. =0.60; Max. =0.95), was als Hinweis auf eine insgesamt befriedigende Reliabilität der gemittelten Wahrnehmungen gewertet werden kann.

Zur Überprüfung der Faktorenstruktur wurde auf Klassenebene eine Faktorenanalyse nach der Hauptkomponentenmethode mit anschliessender Varimax-Rotation durchgeführt ${ }^{1}$. Als Kriterium für die Extraktion eines Faktors diente neben dem Scree-Test und der Interpretierbarkeit der Faktoren die Grösse der Eigenwerte, die nach dem Parallelanalyse-Kriterium (Enzmann, 1997; Lautenschlager, 1989) auf statistische Signifikanz untersucht wurde. Um abzuschätzen, ob empirisch errechnete Eigenwerte statistisch bedeutsam sind oder auf Zufall beruhen können, hat Lautenschlager (1989) umfassende Analysen mit Zufallsdaten unternommen und dabei für eine bestimmte Anzahl von Versuchspersonen und Items berechnet, wie hoch die Eigenwerte nicht-zufälliger Faktoren sein müssen (Parallelanalyse-Kriterium). Das Computerprogramm RanEigen (Enzmann, 1997) verwendet eine lineare Interpolation der von Lautenschlager (1989) bereitgestellten Zufallstabellen, um für eine bestimmte Anzahl von Items und Versuchsteilnehmern diejenigen Faktor- 
eigenwerte $\mathrm{zu}$ berechnen, die noch unter der Zufallsbedingung resultieren können.

Bivariate Zusammenhänge wurden mit einfaktoriellen ANOVA-Analysen gerechnet. Prädiktive Aussagen zwischen fachdidaktischem Handeln und Drittvariablen stützen sich auf Befunde aus Mehrebenenanalysen, die mit dem Computerprogramm HLM 6 (Raudenbush, Bryk, Cheong, Congdon \& du Toit, 2004) durchgeführt wurden. In Untersuchungen mit Schulklassen ist die hierarchische Struktur der gewonnenen Daten zu berücksichtigen, um mögliche Fehlschlüsse bei der Analyse zu vermeiden (Raudenbush \& Bryk, 2002). Aufgrund der hierarchischen Datenstruktur sowie der simultanen Modellierung der Effekte individueller Merkmale (z.B. individuelle Testleistung) bzw. der Schulklasse (z.B. Schulform und fachdidaktische Dimensionen) verbietet sich die konventionelle lineare Regressionsanalyse zur Hypothesenprüfung (Raudenbush \& Bryk, 2002). Ein adäquates Auswertungsverfahren für Daten mit hierarchischer Struktur stellt die Mehrebenenanalyse dar, eine spezielle Form der Regressionsanalyse, die es erlaubt, simultan Prädiktoren auf mehreren Ebenen (z.B. Individualebene, Klassenebene) zu berücksichtigen. Alle berichteten Modelle sind sogenannte random intercept Modelle, die über das restricted maximum likelihood Verfahren geschätzt wurden.

Die gängigen Softwarepakete für Mehrebenenanalysen, wie das von uns verwendete Programm HLM 6, bieten allein unstandardisierte Regressionskoeffizienten in den Ergebnisausdrucken an, was die Interpretation der Regressionsgewichte aufgrund der oftmals arbiträren Metriken der Prädiktoren und Kriterien erschwert. Um die spätere Interpretation zu vereinfachen, wurden in der vorliegenden Arbeit alle metrischen Individual- und Klassenebene-Variablen $z$-standardisiert $(M=0 ; S D=1)$. Die entsprechenden Koeffizienten können daher annähernd im Sinne standardisierter Regressionskoeffizienten interpretiert werden.

Für die Interpretation des hier besonders interessierenden Effekts der fachdidaktischen Herangehensweise haben wir in Anlehnung an jüngere Vorschläge ein einfach interpretierbares Effektstärkenmass herangezogen. Dieses von Tymms (2004; für eine Anwendung vgl. Sammons et al., 2004) vorgestellte Effektstärkenmass für kontinuierliche Level-2-Variablen in Mehrebenenanalysen ist an Cohens $d$-Wert-Mass angelehnt und berechnet sich nach der folgenden Formel:

$$
\Delta=2 \times B \times S D_{\text {predictor }} / \sigma_{\mathrm{e}}
$$

bei der $B$ das unstandardisierte Regressionsgewicht aus dem Mehrebenenmodell ist, $S D_{\text {predictor }}$ die Standardabweichung der Prädiktorvariable auf der Klassenebene $^{2}$ und $\sigma_{\mathrm{e}}$ die Residualstandardabweichung der Outcome-Variable auf der Individualebene. Dies soll an einem kleinen Beispiel demonstriert werden. Gegeben ist ein Regressionskoeffizient auf Klassenebene von $B=.30$, eine Standardabweichung der Prädiktorvariable von $S D=0.35$ und eine Residualstandardabweichung von $\sigma_{\mathrm{e}}=0.80$; es resultiert folgende Gleichung: 


$$
\Delta=2 \times 0.30 \times 0.35 / 0.80=0.26 \text {. }
$$

Diese Effektstärke von $\Delta=0.26$ würde bedeuten, dass sich der Unterschied in der abhängigen Variable zwischen zwei Klassen, die sich in der Prädiktorvariable um zwei Standardabweichungen unterscheiden, auf 0.26 belaufen würde. Nach Cohen's (1988) Konventionen würde dies einen kleinen Effekt darstellen. Angesichts dessen, dass Effektstärken in der Unterrichtsforschung insgesamt eher klein ausfallen und davon auszugehen ist, dass die Effekte über die Jahre eine kumulative Wirkung haben können, halten wir auch kleine Effekte für praktisch bedeutsam.

\section{Ergebnisse}

\section{Faktorielle Struktur}

Explorative Hauptkomponentenanalysen wurden mit dem Programm SPSS gerechnet. Nach dem Parallelanalysekriterium (Lautenschlager, 1989) ergibt sich im vorliegenden Fall bei 30 Items und 92 Klassen ein Verlauf der Zufalls-Eigenwerte von 2.26, 2.06, 1.92, 1.79, 1.68 und 1.59 für die ersten sechs Hauptkomponenten. Es resultierten insgesamt sechs Faktoren, deren Eigenwert grösser als 1 ist. Der empirisch ermittelte Eigenwertverlauf war 8.0, 2.8, 2.7, 2.0, 1.7 und 1.1. Somit lagen lediglich die Eigenwerte der ersten fünf Hauptkomponenten über den Zufalls-Eigenwerten. Auch der Screetest sowie die Interpretierbarkeit der Faktorenlösung liess es angeraten erscheinen, fünf Faktoren zu extrahieren. Die erklärte Varianz der Fünffaktorenlösung betrug 65 Prozent. Die Items und ihre Faktorenladungen sind im Anhang aufgeführt. Alle Items wiesen substanzielle Ladungen auf ihren Primärfaktoren auf. Die Nebenladungen waren mehrheitlich gering. Lediglich in drei Fällen lagen die Koeffizienten merklich über .30 (siehe Tab. im Anhang). Die extrahierten Faktoren stützen mit einer Ausnahme zentrale Annahmen des theoretischen Rahmenmodells.

Faktor I kann in Übereinstimmung mit den theoretischen Vorannahmen als «Unterrichtssprache (Kommunikation) Französisch» (8 Items) umschrieben werden. Auffällig ist, dass sowohl Items der Lehrer-Schüler- als auch der Schüler-Schüler-Interaktion auf demselben Faktor laden. Die Items zur Verwendung der Unterrichtssprache Deutsch laden negativ auf dem Faktor.

Faktor II, «Alltagsgegenstände» genannt (4 Items), betrifft den Gebrauch authentischer Materialien, die einen Bezug zur anderen Sprachkultur bzw. zum ausserschulischen Alltag herstellen können.

Faktor III, als «Übungsintensität» bezeichnet (5 Items), beinhaltet sowohl BICS-Anforderungen als auch automatisierte Übungen, die nur einen eingeschränkten Bezug zu einer konkreten sprachlichen Situation aufweisen. Entgegen unserer Erwartungen differenzierten die Schülerinnen und Schüler bei relativ einfachen Aufgaben somit nicht zwischen kontextualisierten (z.B. eigene 
kleine Texte verfassen) und dekontextualisierten Anforderungen (z.B. Einsetzübungen und Ergänzungstexte).

Faktor IV, «Vokabellernen» genannt (4 Items), umfasst im Wesentlichen drillartiges Lernen von Wortgleichungen, das beim Wortschatzerwerb nach wie vor eine gängige Praxis zu sein scheint. Es handelt sich dabei um dekontextualisiertes Wahrnehmen und Abrufen und betrifft Valette und Disick's (1972) Stufen I und II.

Faktor V, den wir als «Einsatz im wirklichen Leben» (3 Items) bezeichneten, umfasst kommunikative Transferkontakte zu Mitgliedern der anderen Sprachkultur. Auffällig ist, dass Handlungen in diesem Feld III (kontextualisierte, eher anspruchsvolle Aufgaben) in authentischen Kontexten zu erbringen sind. Items, die Simulationen (Rollenspiele) oder die selbstständige Produktion von Texten verlangen und in unserer Konzeption einen Bestandteil dieser Dimension darstellten, waren hingegen zu eliminieren.

\section{Deskriptive Analysen}

Tabelle 1 beinhaltet Verteilungsstatistiken und die Reliabilitätskoeffizienten (Cronbach's Alpha) der einzelnen Skalen auf Klassenebene.

\section{Tabelle 1: Verteilungswerte und interne Konsistenzen der Skalen (Klassenebene)}

\begin{tabular}{|lccccc|}
\hline Skala & M & SD & Min. & Max. & Alpha \\
\hline Unterrichtssprache Französisch (8 Items) & 3.56 & .52 & 2.23 & 4.95 & .92 \\
Authentisches Material (4 Items) & 2.77 & .51 & 1.52 & 4.44 & .88 \\
Übungsintensität (5 Items) & 3.48 & .34 & 2.53 & 4.44 & .75 \\
Vokabeln lernen (4 Items) & 3.43 & .42 & 2.23 & 4.21 & .77 \\
Gebrauch im wirklichen Leben (3 Items) & 1.90 & .70 & 1.08 & 4.48 & .75 \\
\hline
\end{tabular}

Die drei Skalen «Unterrichtssprache Französisch», «Übungsintensität» und «Vokabeln lernen» streuten leicht oberhalb der theoretischen Skalenmitte. Etwas unterhalb der Skalenmitte von 3.0 lag der Mittelwert für die Berücksichtigung authentischer Materialien $(M=2.77, S D=.51)$. Hinsichtlich des Gebrauchs des Französischen im wirklichen Leben fand sich erwartungsgemäss der niedrigste Mittelwert $(M=1.9, S D=.70)$. Auffällig ist hier die beträchtliche Differenz zwischen dem Minimal- und dem Maximalwert. Cronbachs Alpha als Mass für die interne Konsistenz streute zwischen .92 und .75. Hoch ausgefallen sind die Werte für die Faktoren «Unterrichtsprache Französisch» und den Einbezug authentischen Materials. Die Werte für die restlichen drei Faktoren waren zufriedenstellend. Ausser dem Faktor «Gebrauch des Französischen im wirklichen Leben» waren alle Faktoren normal verteilt. 
Tabelle 2: Mittelwerte und Standardabweichungen fachdidaktischer Maßnahmen der Lehrkräfte nach Schulform (Klassenebene)

\begin{tabular}{|lcccc|}
\hline & \multicolumn{2}{c}{$\begin{array}{c}\text { Grund- } \\
\text { ansprüche }\end{array}$} & \multicolumn{2}{c|}{ Erweiterte Ansprüche } \\
& \multicolumn{2}{c}{$\mathrm{N}=37$} & & \multicolumn{2}{c|}{$\mathrm{N}=59$} \\
\hline & $\mathrm{M}$ & $\mathrm{SD}$ & $\mathrm{M}$ & $\mathrm{SD}$ \\
\hline Unterrichtssprache Französisch (8 Items) & 3.34 & .51 & 3.70 & .50 \\
Authentisches Material (4 Items) & 2.72 & .44 & 2.80 & .57 \\
Übungsintensität (5 Items) & 3.47 & .38 & 3.49 & .30 \\
Vokabeln lernen (4 Items) & 3.59 & .33 & 3.34 & .44 \\
Gebrauch im wirklichen Leben (3 Items) & 1.78 & .56 & 1.97 & .78 \\
\hline
\end{tabular}

Tabelle 2 berichtet Mittelwerte und Standardabweichungen für die beiden unterschiedenen Schulformen bzw. Niveaus. In Schulklassen mit erweiterten Ansprüchen wurde im Unterricht mehr französisch gesprochen $(F(1,95)=$ $11,11 ; p=.001)$. In Klassen mit Grundansprüchen lag hingegen der Wert für das Lernen von Vokabeln höher $(F(1,95)=8.52 ; p=.004)$. Nicht statistisch signifikant blieb der Unterschied beim Gebrauch der Sprache im wirklichen Leben. Annähernd gleiche Mittelwerte wiesen die beiden Niveaus hinsichtlich der Übungsintensität und bei der Verwendung authentischer Materialien auf.

Ein einheitliches Bild ergab sich hinsichtlich der Kantonszugehörigkeit. Bei keiner der fünf untersuchten Dimensionen fand sich ein statistisch signifikanter Unterschied.

\section{Korrelative Zusammenhänge $z w i s c h e n$ fachdidaktischen Qualitätsmerkmalen}

In Tabelle 3 sind die Interkorrelationen zwischen den Faktoren sowie ihre Korrelationen mit den Angaben zur Qualität der erteilten Hausaufgaben wiedergegeben. Für die Qualität der Hausaufgaben standen sowohl Angaben der betroffenen Schülerinnen und Schüler als auch externe Beobachterurteile zur Verfügung.

Statistisch signifikante Interkorrelationen von $r=.41$ und $r=.46$ stellten sich zwischen den Faktoren Unterrichtssprache Französisch, Verwendung authentischer Materialien und der Übungsintensität ein. Deutlich geringer mit je $r=.27$ fielen die signifikanten Korrelationen zwischen dem Vokabellernen und der Übungsintensität sowie dem Einsatz des Französischen im wirklichen Leben und der Verwendung authentischer Materialien aus.

Der Gebrauch des Französischen als Unterrichtsprache, die Verwendung authentischen Materials und die Übungsintensität korrelierten in Übereinstimmung mit den hypothetischen Annahmen positiv und statistisch signifikant mit den Urteilen der Schülerinnen und Schüler zur Hausaufgabenqualität. Bei den externen Expertenurteilen traf dies in einem vergleichbaren Ausmasse nur für die 
Korrelation zwischen dem eingeschätzten Anspruchsniveau der Hausaufgaben und der Verwendung der französischen Sprache im Unterricht zu. Geringere, aber durchweg statistisch signifikante Korrelationen ergaben sich auch zwischen den drei Expertenratings und der Verwendung authentischer Materialien.

Tabelle 3: Interkorrelationen der Fachdidaktikfaktoren und Korrelationen zu den Urteilen zur Qualität der Hausaufgaben (Klassenebene)

\begin{tabular}{|c|c|c|c|c|c|c|c|c|}
\hline & (1) & (2) & (3) & (4) & (5) & (6) & (7) & (8) \\
\hline \multicolumn{9}{|l|}{$\begin{array}{l}\text { (1) Unterrichtssprache } \\
\text { Französisch (8 Items) }\end{array}$} \\
\hline $\begin{array}{l}\text { (2) Authentisches Material } \\
\text { (4 Items) }\end{array}$ & $.46^{* * *}$ & & & & & & & \\
\hline $\begin{array}{l}\text { (3) Übungsintensität } \\
5 \text { Items) }\end{array}$ & $.46^{* * *}$ & $.41^{* * *}$ & & & & & & \\
\hline $\begin{array}{l}\text { (4) Vokabeln lernen } \\
\text { (4 Items) }\end{array}$ & .16 & .04 & $.27^{*}$ & & & & & \\
\hline $\begin{array}{l}\text { (5) Einsatz im wirklichen } \\
\text { Leben, Transfer (3 Items) }\end{array}$ & .08 & $.27^{*}$ & .07 & -.02 & & & & \\
\hline $\begin{array}{l}\text { (6) Anspruchsniveau } \\
\text { (Expertenrating) }\end{array}$ & $.42^{* * *}$ & $.37^{* * *}$ & .17 & -.00 & .03 & & & \\
\hline $\begin{array}{l}\text { (7) Einbettung in Lernpro- } \\
\text { zess (Expertenrating) }\end{array}$ & .14 & $.23^{*}$ & .03 & -.08 & .06 & $.61^{* * *}$ & & \\
\hline $\begin{array}{l}\text { (8) Lebensweltlicher Bezug } \\
\text { (Expertenrating) }\end{array}$ & .19 & $.28^{*}$ & .07 & .11 & .05 & $.53^{* * *}$ & $.60^{* * *}$ & \\
\hline (9) Schülerurteile & $.44^{* * 3}$ & $.58^{* * *}$ & $.58^{* * *}$ & -.00 & .03 & .16 & .19 & .11 \\
\hline
\end{tabular}

Anmerkung: ${ }^{*} \mathrm{p}<.05 ;{ }^{* * *} \mathrm{p}<.001$

\section{Korrelationen mit den Zielkriterien}

Berücksichtigt man zusätzlich die Korrelationen der beiden Zielkriterien Interesse und Leistung, dann stellt man in Tabelle 4 fest, dass die Zusammenhänge zwischen dem ersten und zweiten Messzeitpunkt sehr hoch ausgefallen sind. Dies betrifft insbesondere die Französischleistung. In einem mittleren Bereich lagen hingegen die Werte zwischen Leistung und Interesse.

Tabelle 4: Mittelwerte, Standardabweichungen sowie Interkorrelationen und Korrelationen der Zielkriterien zwischen den beiden Messzeitpunkten (Klassenebene)

\begin{tabular}{|lccccc|}
\hline & $\mathrm{M}$ & $\mathrm{SD}$ & $(1)$ & $(2)$ & $(3)$ \\
\hline (1) Interesse t1 & 2.76 & .24 & & & \\
(2) Interesse t2 & 2.67 & .26 & $.77^{* * *}$ & & \\
(3) Leistung t1 & -.14 & .83 & $.43^{* * *}$ & $.46^{* * *}$ & \\
(4) Leistung t2 & .25 & 1.06 & $.41^{* * *}$ & $.49^{* * *}$ & $.94^{* * *}$ \\
\hline
\end{tabular}

Anmerkung: ${ }^{* * *} \mathrm{p}<.01$ 
Tabelle 5: Korrelationen der Unterrichtsmerkmale mit den Zielkriterien (Klassenebene)

\begin{tabular}{|lcccc|}
\hline & Interesse t1 & Interesse t2 & Leistung t1 & Leistung t2 \\
\hline Unterrichtssprache Französisch & $.40^{* * *}$ & $.52^{* * *}$ & $.28^{* *}$ & $.33^{* *}$ \\
Authentisches Material & $.38^{* * *}$ & $.23^{*}$ & -.04 & -.02 \\
Übungsintensität & $.38^{* * *}$ & $.52^{* * *}$ & .10 & .17 \\
Vokabeln lernen & -.01 & -.01 & $-.35^{* * *}$ & $-.31^{* *}$ \\
Einsatz im wirklichen Leben & .09 & -.06 & .14 & -.02 \\
\hline
\end{tabular}

Anmerkung: ${ }^{*} \mathrm{p}<.05 ;{ }^{* *} \mathrm{p}<.01 ;{ }^{* * *} \mathrm{p}<.001$

Werden diese Zielkriterien in Tabelle 5 zudem mit den fachdidaktischen Unterrichtsmerkmalen korreliert, dann fällt vorerst ein durchgehender statistisch bedeutsamer Zusammenhang zwischen dem Gebrauch der Unterrichtssprache Französisch und den beiden Zielkriterien auf. Die Verwendung authentischen Materials sowie die Übungsintensität korrelierten jedoch nur mit dem Interesse am Fach. Das Lernen von Vokabeln stand dagegen in einer negativen Beziehung zur Leistung. Gemäss Tabelle 2 ist dabei allerdings zu beachten, dass Schülerinnen und Schüler aus Schulklassen mit Grundansprüchen über mehr Vokabellernen berichteten. Diese Zusammenhänge können in den folgenden Vorhersageanalysen differenzierter untersucht werden.

\section{Vorhersage von Leistung und Interesse}

Während die bisher berichteten Analysen ausschliesslich auf der Ebene von Klassen durchgeführt wurden, handelt es sich bei den nachfolgend berichteten Berechnungen um Mehrebenenanalysen, in denen neben der Ebene der Schulklassen auch die Ebene der Schülerinnen und Schüler berücksichtigt wurde. Für die beiden abhängigen Variablen Leistung zu t2 und Interesse zu t2 wurden je sieben Modelle spezifiziert. Auf der individuellen Ebene wurden die beiden Prädiktoren Geschlecht und Leistung zu t1 bzw. Interesse zu t1 in allen Modellen als Kontrollvariablen unzentriert eingeführt. Auf der Klassenebene wurden die beiden strukturellen Variablen Kanton (zwei Dummy-Variablen) und Schulform (eine Dummy-Variable) in allen Modellen berücksichtigt. Da zwischen den fachdidaktischen Dimensionen teilweise ein bedeutsamer Zusammenhang bestand, wurde in den Modellen 2 bis 6 jeweils nur ein fachdidaktischer Prädiktor berükksichtigt. In Modell 7 wurden schliesslich alle fachdidaktischen Prädiktoren simultan in die Berechnungen einbezogen. 


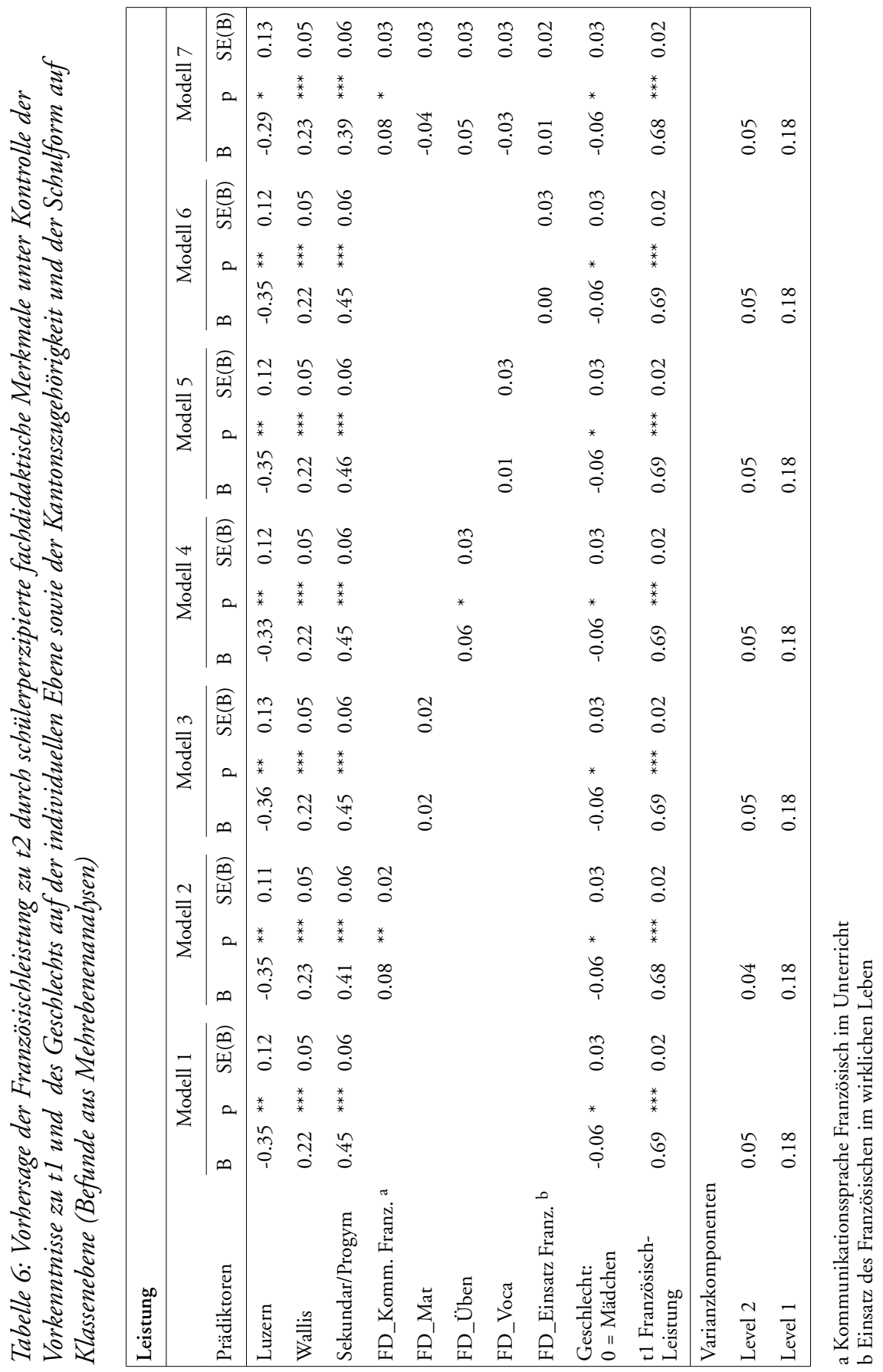


In Modell 1 zur Vorhersage der Leistung zum zweiten Messzeitpunkt (vgl. Tab. 6) zeigten Jungen im Vergleich mit den Mädchen bei Kontrolle der anderen berücksichtigten Variablen geringere Leistungen $(B=0.06)$. Erwartungsgemäss war der prädiktive Effekt der Französischleistung zum Zeitpunkt t1 hoch bedeutsam $(B=0.68)$. Dies weist nachdrücklich auf die Bedeutung des Vorwissens bei der Erklärung der Leistung zum zweiten Messzeitpunkt hin. Signifikante Effekte resultierten auch für die Kantonszugehörigkeit und für die Schulform. Im Vergleich zur Referenzgruppe der Freiburger Schülerinnen und Schüler zeigten Lernende aus dem Kanton Luzern bei Kontrolle der anderen Variablen statistisch signifikant geringere Leistungen $(B=-0.35)$. Hingegen war die Zugehörigkeit zum Kanton Wallis bei Kontrolle der übrigen Variablen (inklusive der Leistung zu t1) mit einer im Vergleich zum Kanton Freiburg höheren Leistung assoziiert $(B=0.22)$.

In den Modellen 2 bis 6 wurden prädikative Effekte der einzelnen fachdidaktischen Komponenten geprüft. Wie aus Tabelle 6 hervorgeht, konnten die postulierten Zusammenhänge für die Skalen «Unterrichtssprache Französisch» sowie «Übungsintensität» bestätigt werden. Rechnet man den Effekt für die Unterrichtssprache $(B=.08)$ nach der oben beschriebenen Formel zur Berechnung von Effektstärken in Mehrebenenanalysen um, so beträgt die Effektstärke $\Delta=$.36; bei dem Effekt für die Unterrichtssprache handelt es sich demnach um einen kleinen, aber durchaus bedeutsamen Effekt. Die Effektstärke für die Variable Übungsintensität betrug $\Delta=.29$. Für die drei übrigen fachdidaktischen Variablen (Schaffung authentischer Kommunikationsmöglichkeiten, Einbezug authentischer Materialien und Vokabellernen) konnten keine statistisch signifikanten Effekte gefunden werden.

Abschliessend wurden in Modell 7 alle fünf fachdidaktischen Prädiktoren simultan berücksichtigt. Dabei zeigte sich, dass bei Kontrolle aller Variablen lediglich der Faktor «Unterrichtssprache Französisch» einen statistisch signifikanten Effekt hatte. Seine Effektstärke stieg dabei auf $\Delta=.38$ an. Die Bedeutsamkeit dieses Faktors wurde damit bestätigt. Der Faktor «Übungsintensität» hatte dagegen keinen statistisch signifikanten Effekt in Modell 7; dies dürfte massgeblich auf den relativ engen Zusammenhang mit dem Faktor «Unterrichtssprache Französisch» (vgl. Tab. 3) zurückgeführt werden können.

Ein ähnliches Bild wie die Analysen zur Vorhersage der Leistung zu t2 ergaben die Analysen mit der abhängigen Variable «Interesse am Fach Französisch». In Modell 1 zur Vorhersage des Interesses zum zweiten Messzeitpunkt zeigten Jungen im Vergleich mit den Mädchen bei Kontrolle der anderen berücksichtigten Variablen ein signifikant geringeres Interesse $(B=0.15)$. Wiederum war der prädiktive Effekt des Interesses zum Zeitpunkt t1 erheblich $(B=0.65)$; hierin drückt sich die Stabilität des Interesses im Laufe eines Schuljahres aus. Im Vergleich zur Referenzgruppe der Freiburger Schülerinnen und Schüler zeigten Schüler aus dem Kanton Wallis bei Kontrolle der anderen Variablen signifikant mehr Interesse $(B=.13)$. Derselbe Effekt resultierte auch für Schüler der beiden höheren Schulformen Sekundarschule und Progymnasium $(B=.19)$. 


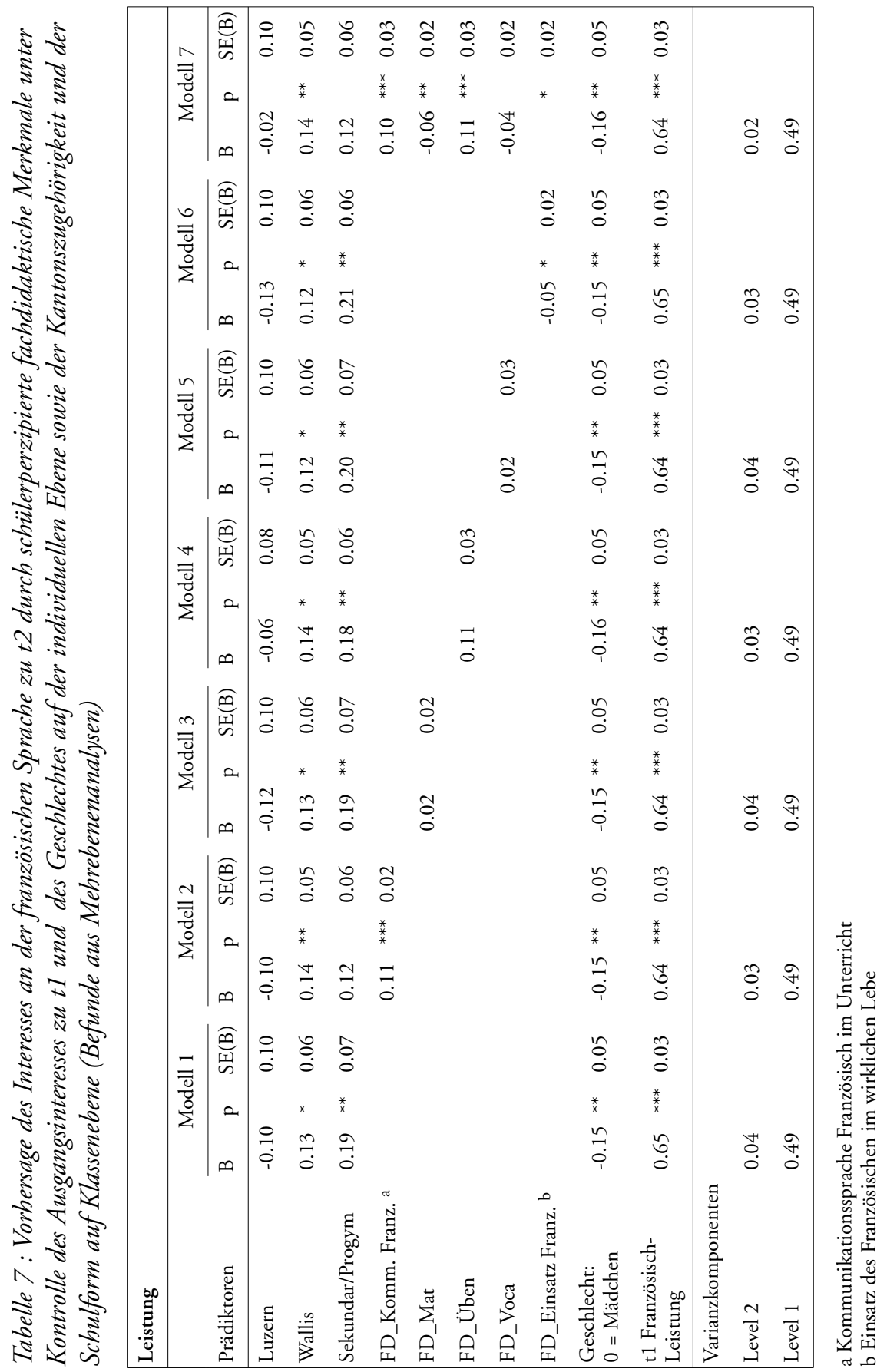


In den Modellen 2 bis 6 erwiesen sich die beiden Faktoren «Unterrichtssprache Französisch» $(\Delta=.32)$ und «Übungsintensität» $(\Delta=.33)$ auch für den Anstieg des Interesses als statistisch signifikant und bedeutsam. Diese Effekte blieben auch in Modell 7, in dem wiederum alle fachdidaktischen Variablen simultan berücksichtigt wurden, praktisch konstant. Der Einsatz des Französischen im Austausch mit Fremdsprachigen schien hingegen das Interesse an der Sprache eher negativ zu beeinflussen. Dieser erwartungswidrige Effekt war mit $\Delta=.14$ zwar sehr klein, aber dennoch statistisch signifikant. Auffällig war zudem, dass die Hinzunahme authentischer Materialien bei Kontrolle aller Variablen nunmehr ebenfalls einen statistisch signifikant negativen Effekt ergab. Allerdings war auch dieser Effekt mit $\Delta=.18$ recht klein.

\section{Diskussion}

\section{Fachdidaktische Dimensionen}

Die Qualität der wahrgenommenen Lernaktivitäten im Fremdsprachenunterricht konnte durch die beiden in Abbildung 1 dargestellten Dimensionen «Grad der kognitiven Ich-Beteiligung» und "Zunehmende Dekontextualiserung» für die folgenden beiden Faktoren bestätigt werden: Reine Feld II-Anforderungen (dekontextualisertes Automatisieren - einfache kognitive Anforderungen) konnten erstens für das Vokabellernen extrahiert werden. Dies ist nicht weiter erstaunlich. Beim Wortschatzerwerb ist das Lernen von Vokabelgleichungen (Abdecken von Wortlisten) eine nach wie vor sehr verbreitete Praxis mit wohl prägender Wirkung auf die Wahrnehmung der eigenen, alltäglichen Lernerfahrungen. Ein weiterer Faktor mit Indikatoren für das Transferfeld III (Bewältigung kognitiv komplexer kontextbezogener Anforderungen) beschränkt sich auf Items, die Kontakte mit der anderen Sprachkultur erfassen. Auch dabei handelt es sich um einen funktional klar begrenzten Erfahrungsausschnitt. Ein etwas breiteres Spektrum sprachlicher Lernaktivitäten repräsentiert hingegen der Faktor «Übungsintensität». Die fraglichen Items stammen sowohl aus Feld I wie auch aus Feld II. Bei den dazu passenden Aufgaben handelt es sich somit um einen Mix aus kontextualisierten und dekontextualisierten Anforderungen im unteren Stufenbereich kognitiver Ich-Beteiligung. Die Kontextualisierungsdimension erwies sich für diesen Faktor damit nicht mehr als relevant. Insgesamt beinhaltet er jedoch variierte Opportunitäten des Übens und Anwendens, die auch häufig genannten Kriterien der Unterrichtsqualität genügen (Brophy \& Good, 1986).

Beim kommunikativen Gebrauch der Zielsprache im Unterricht schien es unerheblich zu sein, ob die Lernenden untereinander oder mit der Lehrperson in frontalen Phasen fremdsprachlich kommunizierten: Sämtliche Items der fremdsprachlichen Kommunikation waren in einem einzelnen Faktor vereinigt. Die Korrelation dieser fremdsprachlichen Kommunikation mit dem Faktor 
«Übungsintensität» lag in einem mittleren Bereich. Dieser Befund ist plausibel, da intensives Üben mit dem Gebrauch der Fremdsprache kovariiert. Auch der Einbezug authentischer Zusatzmaterialien besitzt in der Wahrnehmung der Schülerinnen und Schüler eine eigene Qualität.

Die Ergebnisse machen deutlich, dass die Schülerinnen und Schüler zwischen fachdidaktischen Erfahrungsfeldern differenzieren, die einerseits auf typische Aufgabenfelder fokussiert sind, andererseits aber auch mit persönlichen Erfahrungsqualitäten assoziiert werden. Einschränkend ist natürlich hervorzuheben, dass ihnen mit 30 Items zwar ein ausführlicher Fragenkatalog vorgelegt wurde, der jedoch in keinster Weise als exhaustiv zu bezeichnen ist. Zu überlegen wäre, ob das Instrument noch etwas verfeinert werden könnte und sich einzelne Nebenladungen von Items, die über 0.30 liegen, damit verringern liessen.

\section{Häufigkeit und Zusammenhang zwischen einzelnen Unter- richtsdimensionen}

Betrachtet man die Verteilungs- und die Streuungsmasse der einzelnen Faktoren in Tabelle 1, dann fällt auf, dass die Komponenten, die die Intensität des Unterrichts betreffen (Kommunikation in der Fremdsprache, Übungsintensität, Vokabellernen), um die theoretische Skalenmitte streuen. Demgegenüber konzentrierte sich der Gebrauch der Fremdsprache im Austausch mit Menschen, die diese Sprache sprechen, wohl eher auf einzelne Klassen. Der hohe Maximalwert lässt zudem darauf schliessen, dass sich diese Praxis in bestimmten Schulklassen mit einer gewissen Regelmässigkeit etabliert haben könnte. Die Lehrkräfte schienen sich im Weiteren zu bemühen, neben dem gängigen Lehrmittel auch zusätzliche Materialien aus dem Alltag einzusetzen. Dieses fachdidaktische Anliegen dürfte die Praxis inzwischen auf eine erkennbare Art und Weise erreicht haben.

Analysiert man in der Folge die Unterschiede, die zwischen den einzelnen Schulformen bzw. -niveaus bestanden, dann stellt man fest, dass in Klassen mit Grundansprüchen vergleichsweise weniger französisch gesprochen wurde. Stattdessen mussten anteilsmässig mehr Vokabeln gelernt werden. Was könnten die Gründe für diesen Befund sein? Aufgrund anzunehmender Defizite hinsichtlich kommunikativer Fertigkeiten der Schülerinnen und Schüler in Schulformen mit Grundansprüchen könnten sich die Lehrkräfte möglicherweise geneigt sehen, den Stellenwert des Wortschatzerwerbs zu erhöhen. Aufgrund des im zugrunde liegenden Datensatz nahezu ausgebliebenen Leistungsanstiegs bei Schülerinnen und Schülern mit Grundansprüchen (Neumann et al., 2007) scheint eine Überprüfung der fraglichen Praxis ratsam zu sein, denn die Sprachverwendung wird erst in der Kommunikationssituation wirklich automatisiert (Hulstijn, 1997).

Für die Analyse von Zusammenhängen zwischen fachdidaktischen Ansprüchen und Indikatoren der Hausaufgabenpraxis standen qualitative Ratings sowohl von Schülerinnen und Schülern als auch von externen Expertenpersonen zur Verfügung. Nach den Urteilen der Schülerinnen und Schüler zu schliessen, scheint es so zu sein, dass Lehrerinnen und Lehrer, deren Unterricht sich durch 
eine hohe Übungsintensität auszeichnete, auch mehr Wert auf die Qualität der Hausaufgaben gelegt hatten. Damit hing zusammen, dass diese Lehrkräfte die Fremdsprache im Unterricht häufiger gebrauchten und auch mehr authentische Materialien einsetzten, wozu gerade bei den Hausaufgaben ausreichend Gelegenheit besteht. Lehrpersonen, die nach Angaben ihrer Schülerinnen und Schüler mehr Wert auf den Gebrauch des Französischen im Unterricht gelegt hatten, wurde auch von den Experten ein höheres Anspruchsniveau bei den Hausaufgaben attestiert. Insgesamt zeigt sich dabei ein bemerkenswerter Zusammenhang zwischen der Hausaufgabenvergabe und fachdidaktisch bedeutsamen Praktiken der Lehrkräfte im Unterricht. Die mittelgrossen Korrelationen erlauben den Schluss, dass es sich dabei um Bereiche handelt, die zwar zusammenhängen, aber dennoch eine getrennte Betrachtung verdienen.

\section{Vorhersage von Leistung und Interesse}

Statistisch signifikante positive Effekte auf die Leistung konnten für die beiden Skalen «Unterrichtssprache Französisch» und «Übungsintensität» nachgewiesen werden. Die Bestätigung der entsprechenden Hypothesen erfolgte nach Kontrolle der Leistung zu t1 und des Geschlechtes der Schülerinnen und Schüler auf der individuellen Ebene sowie des Herkunftskantons und der Schulform bzw. des Niveaus auf der Klassenebene. Die simultane Berücksichtigung aller fachdidaktischen Prädiktorvariablen in der Regressionsgleichung hatte jedoch zur Folge, dass der Effekt für den Faktor «Übungsintensität» nicht mehr statistisch signifikant war. Aufgrund des relativ engen Zusammenhangs der beiden Variablen ist dieser Befund allerdings wenig überraschend. Häufiges Üben und Anwenden dürfte in einem gewissen Masse auch mit dem Gebrauch der französischen Sprache durch die Beteiligten kovariieren. Die Korrelation dieser beiden Faktoren (vgl. Tab. 3) spricht für diese Interpretation. Die statistisch signifikanten negativen Korrelationen zwischen dem Vokabellernen und der Leistung in Tabelle 5 konnten in der Mehrebenenanalyse unter Berücksichtigung relevanter Kontrollvariablen auf der Individual- und Klassenebene somit nicht mehr bestätigt werden. Das mehr oder weniger häufige Lernen von Vokabeln blieb für den Leistungsanstieg somit folgenlos. Es mag zwar ein notwendiges Element beim Aufbau des Wortschatzes sein, dennoch scheint es plausibel zu sein, dass die Beherrschung kommunikativer Basisfertigkeiten erst durch Prozesse höherer IchBeteiligung in den Sprachfeldern I und III zustande kommen dürfte (Scherfer, 2003). Die Berücksichtigung authentischer lebensweltlicher Materialien sowie die Schaffung von Austauschmöglichkeiten zur anderen Sprachkultur wirkten sich ebenfalls nicht statistisch signifikant auf die Leistungsgenese innerhalb eines Schuljahres aus. Gemäss den hypothetischen Vorannahmen wurden für diese Komponenten allerdings eher Zusammenhänge mit dem Interesse an der Fremdsprache erwartet.

Die Voraussagen für den Gebrauch der französischen Unterrichtssprache und die Übungsintensität wurden auch für die Entwicklung des Interesses an der 
Fremdsprache bestätigt. Im Unterschied zum festgestellten Zusammenhang mit dem Leistungsanstieg blieb der statistisch signifikante Effekt der Übungsintensität auch bei der simultanen Berücksichtigung aller fachdidaktischen Variablen sowie der strukturellen und individuellen Komponenten nahezu konstant. Es lässt sich spekulieren, dass die Lernenden einen Unterricht, der sich durch diese beiden Merkmale auszeichnet, als lernwirksam wahrnehmen. Diese positive Erfahrung kann sich längerfristig auch günstig auf das Interesse am Fach auswirken. Der Befund, dass teilweise dieselben fachdidaktischen Merkmale mit einer günstigen Entwicklung von Leistung und Interesse assoziiert waren, ist sicherlich von Bedeutung, da er der These einer Inkompatibilität kognitiver und motivationaler Zielkriterien widerspricht. Inwieweit zu diesem Befund auch eine etwaige Reziprozität von Leistung und Interesse (Baumert, Schnabel \& Lehrke, 1998; Köller, Trautwein, Lüdtke et al., 2006; Marsh et al., 2005; Schiefele et al., 1993) beitrug, sollen vertiefenden Analysen mit dem vorliegenden Datensatz klären.

Für den Anstieg des Interesses waren allerdings auch erwartungswidrige prädiktive Effekte feststellbar. Schülerinnen und Schüler aus Klassen, die angaben, im Unterricht auch Kontakte zu Menschen aufgenommen zu haben, welche die Zielsprache sprechen, äusserten - bei Kontrolle des Ausgangsniveaus - am Ende des Jahres ein signifikant geringeres Interesse am Fach im Vergleich mit Schülerinnen und Schülern, in deren Unterricht diese fachdidaktische Strategie eine geringere Rolle spielte. Zwar ist der Effekt eher gering ausgefallen. Dennoch ist das Ergebnis bedeutsam, wenn es zum Aufwand in Beziehung gesetzt wird, der für die Durchführung dieser Austauschkontakte aufgewendet werden muss. Man kann für diesen Effekt möglicherweise mehrere Gründe ins Feld führen. Es könnte sein, dass die Zugangsschwelle für einen derartigen Austausch für manche Schülerinnen und Schüler zu hoch angesetzt worden ist. Der zu bewältigende Grad der Komplexität könnte sie entmutigt haben (Lightbown, 2000). Denkbar ist auch, dass bei der fraglichen Altersgruppe der Jugendlichen Reaktanzphänomene provoziert werden können, weil ihnen kommunikative Beziehungen zugemutet werden, die sie im Alltag üblicherweise autonom eingehen möchten. Damit hängt der Befund zusammen, dass eine Massnahme in der Regel nicht a priori wirksam zu sein braucht. Massgebend dürfte vielmehr die Qualität sein, mit der sie durchgeführt wird (Klieme et al., 2006). So dürfte beispielsweise die Herstellung von Beziehungen zu Menschen, welche die Zielsprache sprechen, gegenseitige Reziprozität der Perspektiven verlangen (Hodel, 2006). Wünschbare pädagogische Voraussetzungen von Austauschmassnahmen hat Saudan (2003) in seiner Studie herausgearbeitet.

Ein erwartungswidriger Effekt war im Weiteren auch bei der Hinzunahme lebensweltlicher Medien bzw. Materialien feststellbar, allerdings nur unter der simultanen Berücksichtigung aller Fachdidaktikkomponenten. Negative Nebenwirkungen könnten eingetreten sein, weil die verwendeten Materialien zu wenig auf die Voraussetzungen der Lernenden abgestimmt gewesen waren. In solchen 
Fällen könnten unter Umständen Erwartungseffekte des Nicht-Könnens aufgebaut werden. Lehrmittelunabhängige Materialien entfalteten ihre Wirkung somit nur im Verbund lernwirksamer methodischer Massnahmen. Eine weitere Erklärung für den erwartungswidrigen Effekt könnte sein, dass sich die Schülerinnen und Schüler generell an Gütekriterien des Fremdsprachenunterrichts orientierten, die im Wesentlichen durch ein vorgegebenes Lehrmittel determiniert waren. Abweichungen von diesem Lehrmittel konnten dann als Zeitverschwendung wahrgenommen worden sein, weil sie aus subjektiver Schülersicht den notwendigen Gang des Unterrichts gehemmt hatten.

\section{Zusammenfassung und Ausblick}

Insgesamt sind die Befunde der vorliegenden Studie sowohl von theoretischer als auch von praktischer Bedeutung. Konzeptuell beschäftigte sich die bisherige Unterrichtsforschung vornehmlich mit strukturellen Bedingungen effizienten Unterrichts wie Klarheit, Zeitnutzung und Klassenführung. In jüngeren Ansätzen wird hingegen versucht, die Instruktionsqualität genauer zu erfassen (Greeno, 1998). Man versteht darunter beispielsweise die Inszenierung von fachspezifischen Lernprozessen oder auch die Verwendung bestimmter Aufgabentypen. Dabei wird insbesondere die Bedeutung kognitiv anregender Herausforderungen hervorgehoben (z.B. Kunter \& Baumert, 2006b; Tsai et al., in press). Die Intention der vorliegenden Studie lag ebenfalls darin, spezifische Instruktionsgegebenheiten für den gängigen Fremdsprachenunterricht zu konzeptualisieren und empirisch zu prüfen. Neben instruktionalen Verhaltensweisen wie dem Gebrauch der Unterrichtssprache und der Verwendung authentischer Medien konnten auch drei Aufgabenkategorien bestimmt werden. Bei letzteren ist bemerkenswert, dass sich die spezifische Bedeutung anspruchsvoller Aufgabenstellungen im Fremdsprachenunterricht nicht bestätigt hat. Hier scheinen auch einfache kognitive Anforderungen relevant zu sein, wie sie beispielsweise der Faktor Üben/Anwenden beinhaltet (vgl. dazu auch Klieme et al., 2006). Die vorliegenden Befunde könnten infolgedessen auch dazu Anlass geben, konstruktivistisch begründbare Forderungen nach High-level-Aktivitäten domänen- und adressatenspezifisch zu differenzieren sowie noch genauer zwischen herausfordernden und überfordernden Unterrichtsskripten zu unterscheiden.

Von praktischer Bedeutung sind Informationen über den Status gängiger didaktischer Massnahmen im Unterrichtsalltag und ihre Bedeutung für die Leistung und das Interesse der Schülerinnen und Schüler. Dabei scheinen vor allem Intensitätsfaktoren, wie der Gebrauch des Französischen im Unterricht, sowie häufiges Üben/Anwenden bedeutsam zu sein. Die festgestellten hypothesenwidrigen Befunde zum Interesse an der Fremdsprache könnten zudem als ein Indiz gelten, dass der alleinige Einsatz fachdidaktisch propagierter Methoden nicht a priori wirksam ist. Zugleich muss gewährleistet sein, Massnahmen für eine erfolgreiche Implementation zu ergreifen. Darüber hinaus könnten diese Befunde auch zum Anlass genommen werden, bei der Ermöglichung authentischer Spra- 
cherfahrungen, neben den subjektiven Vorstellungen der Lehrkräfte (Schneider, 2007), auch diejenigen der Schülerinnen und Schüler nicht unbeachtet zu lassen.

\section{Anmerkungen}

1 Unser Beitrag konzentrierte sich auf die Klassenebene und liess die Individualebene aussen vor. Dies ist im vorliegenden Fall angemessen, weil das verwendete Instrument eindeutig auf die Erfassung der Lernumwelt abzielt (Cronbach, 1976, für eine ausführlichere Diskussion). Von daher stand auch die psychometrische Analyse der Items auf Klassenebene im Vordergrund. Wir haben uns hierbei auf Analysen auf Klassenebene gestützt, die mit SPSS durchgeführt wurden. Eine Alternative zu diesem Vorgehen stellt das noch junge Verfahren der explorativen Mehrebenenfaktorenanalyse dar, bei der für die Berechnung der Faktorenstruktur auf Klassenebene auch die Varianzanteile auf Individualebene berücksichtigt werden. Allerdings erschien im vorliegenden Falle die Zahl der Klassen für eine Mehrebenenfaktorenanalyse eher grenzwertig zu sein (vgl. auch Lüdtke, Trautwein, Schnyder \& Niggli, 2007). Auch bestand dezidiert kein Interesse an der Analyse der Individualebenendaten zur Fachdidaktik, da wir hierfür keine theoretischen Vorhersagen hatten. Zudem fallen die Ergebnisse einer konventionellen Faktorenanalyse sowie der explorativen Mehrebenenfaktorenanalyse bei ausreichenden Stichprobengrössen innerhalb der Klassen in der Regel sehr ähnlich aus. Nach Abwägung des potenziellen Mehrwerts einer Mehrebenenfaktorenanalyse und der Konsequenzen in Hinblick auf die Länge des Artikels wurde auf eine Mehrebenenfaktorenanalyse verzichtet.

2 Die Entscheidung, zwei Standardabweichungen zu verwenden, hat Tymms (2004) getroffen. Sie ist letzten Endes etwas willkürlich.

\section{Literaturverzeichnis}

Baumert, J., Schnabel, K.-U., Lehrke, M. (1998). Learning math in school: Does interest really matter? In L. Hoffmann, A. Krapp, K. A. Renninger \& J. Baumert (Eds.), Interest and learning: proceedings of the Seeon conference on interest and gender (pp. 327-336). Kiel: Institut für die Pädagogik der Naturwissenschaften an der Universität Kiel.

Bleyhl, W. (2005). Die Defizite des traditionellen Fremdsprachenunterrichts oder: Weshalb ein Paradigmenwechsel, eine Umkehr im Fremdsprachenunterricht erfolgen muss. Fremdsprachen lehren und lernen, 34 (1), 45-64.

Blömeke, S., Risse, J., Müller, C., Eichler, D. \& Schulz, W. (2006). Analyse der Qualität von Aufgaben aus didaktischer und fachlicher Sicht. Unterrichtswissenschaft, 34 (4), 330-357.

Brophy, J. E. \& Good, T. L. (1986). Teacher behavior and student achievement. In M. C. Wittrock (Ed.), Handbook of research on teaching (pp. 328-375). London: Mc Millan.

Campbell, J., Kyriakides, L., Muijs, D. \& Robinson, W. (2005). Assessing teacher effectiveness. Developing a differentiated model. New York: Routledge Falmer.

Clausen, M. (2002). Qualität von Unterricht-eine Frage der Perspektive? Münster: Waxmann.

Cohen, J. (1988). Statistical power analysis for the behavioral sciences (2nd ed.). Hillsdale, NJ: Erlbaum.

Cronbach, L. J. (1976). Research on classrooms and schools: Formulations of questions, design and analysis. Stanford, CA: Stanford Evaluation Consortium.

Cummins, J. (1979). Cognitive-academic language proficiency. Linguistic interdependence, the optimum age question and some other matters. Working papers on bilinguism, 19, 197-205.

Cummins, J. (2001). Language, power and pedagogy. Bilingual children in the crossfire. Clevedon: Multilingual Matters.

Eccles, J. S. \& Wigfield, A. (2002). Motivational beliefs, values and goals. Annual Review of Psychology, 53, 109-132. 
Edelstein, W. (1970). Das Projekt Schulleistung am Max-Planck-Institut für Bildungsforschung. Zeitschrift für Pädagogik, 16 (4), 517-529.

EDK (2004). HarmoS - Zielsetzung und Konzeption. Bern: EDK.

Edmondson, W. \& House, J. (2000). Einführung in die Sprachlehrforschung. Tübingen: Francke.

Ellis, R. (2005). Instructed second language acquisition: A literature review. Ministry of Education, New Zealand. [http://www.educationcounts.edcentre.govt.nz/publications/ downloads/instructed-second-language.pdf]. (12.07.2007)

Enzmann, D. (1997). RanEigen: A program to determine the parallel analysis criterion for the number of principal components. Applied Psychological Measurement, 21, 232.

Finkbeiner, C. (2001). Untersuchungsfeld 2: Attidudionale und affektive Aspekte. In H. J. Vollmer, G. Henrici, C. Finkbeiner, R. Grotjahn, G. Schmid-Schönbein \& W. Zydatiss (Hrsg.), Lernen und Lehren von Fremdsprachen: Kognition, Affektion, Interaktion. Ein Forschungsüberblick. Zeitschrift für Fremdsprachenforschung, 12 (2), 65-75.

Finkbeiner, C. (2003). Zur Evaluation von Fremdsprachenunterricht und fremdsprachlicher Leistung. Empirische Pädagogik, 17 (3), 295-311.

Grau, M. \& Würffel, N. (2003). Übungen zur interkulturellen Kommunikation. In K. R. Bausch, H. Christ \& H.-J. Krumm (Hrsg.), Handbuch Fremdsprachenunterricht (S. 312314). Tübingen und Basel: A. Francke UTB.

Greeno, J. G. and the Middle School Mathematics Through Applications Project Group. (1998). The situativity of knowing, learning and research. American Psychologist, 53 (1), 5-26.

Grin, F. (1999). Compétences linguistiques en Suisse: Bénéfices privés, bénéfices sociaux et dépenses. Bern, Aarau: Schweizerische Koordinationsstelle für Bildungsforschung, SKBF.

Gruehn, S. (2000). Unterricht und schulisches Lernen. Münster: Waxmann.

Haudeck, H. (2005). Wie "pauken» Schüler und Schülerinnen Vokabeln für den Fremdsprachenunterricht? In P. Mayring \& M. Gläser-Zikuda (Hrsg.), Die Praxis der Qualitativen Inhaltsanalyse (S. 84-104). Weinheim und Basel: Beltz Verlag.

Helmke, A. \& Schrader, F. W. (2001). Determinanten der Schulleistung. In D. H. Rost (Hrsg.), Handwörterbuch Pädagogische Psychologie (S. 81-91). Weinheim: Beltz.

Helmke, A. \& Schrader, F. W. (2002). Jenseits von TIMSS: Messungen sprachlicher Kompetenzen, komplexe Längsschnittstudien und kulturvergleichende Analysen. In F. E. Weinert (Hrsg.), Leistungsmessungen in Schulen (S. 237-250). Weinheim: Beltz.

Helmke, A. \& Weinert, F. E. (1997). Bedingungsfaktoren schulischer Leistungen. In F. E. Weinert (Hrsg.), Enzyklopädie der Psychologie: Bd. 3. Psychologie der Schule und des Unterrichts (S. 71-176). Göttingen: Hogrefe.

Hidi, S. \& Renninger, K. A. (2006). The four-phase model of interest development. Educational Psychologist, 41 (2), 111-127.

Hodel, H. P. (2006). Sprachaufenthalte. Perspektiven und Untersuchungen im ausserschulischen Sprachenlernen. Tübingen und Basel: Francke.

Hulstijn, J. (1997). Mnemonic methods in foreign language vocabulary learning. In J. Coady \& T. Huckin (Eds.), Second language vocabulary acquisition (pp. 203-224). Cambridge: Cambridge University Press.

Judd, E. L., Tan, L. \& Walberg, H. J. (2001). Teaching additional languages. Geneva: International Academy of Education - International Bureau of Education.

Klieme, E., Eichler, W., Helmke, A., Lehmann, R. H., Nold, G., Rolff, H. G., Schröder, K., Thomé, G. \& Willenber, H. (2006). Unterricht und Kompetenzerwerb in Deutsch und Englisch. Zentrale Befunde der Studie Deutsch Englisch Schülerleistungen International (DESI) . Frankfurt/M.: Deutsches Institut für Internationale Pädagogische Forschung (DIPF).

Köller, O., Baumert, J., Cortina, K., Trautwein, U. \& Watermann, R. (2004). Öffnung von Bildungswegen in der Sekundarstufe II und die Wahrung von Standards. Zeitschrift für Pädagogik, 50 (5), 679-700.

Köller, O. \& Trautwein, U. (2004). Englischleistungen von Schülerinnen und Schülern an all- 
gemein bildenden und beruflichen Gymnasien. In O. Köller, R. Watermann, U. Trautwein \& O. Lüdtke (Hrsg.), Wege zur Hochschulreife in Baden-Württemberg. TOSCA - Eine Untersuchung an allgemein bildenden und beruflichen Gymnasien (S. 285-326). Opladen: Leske und Budrich.

Köller, O., Trautwein, U., Cortina, K. \& Baumert, J. (2006). Rezeptive Kompetenzen in Englisch am Ende der gymnasialen Oberstufe. Unterrichtswissenschaft, 34 (1), 239-255.

Köller, O., Trautwein, U., Lüdtke, O. \& Baumert, J. (2006). Zum Zusammenspiel von schulischer Leistung, Selbstkonzept und Interesse in der gymnasialen Oberstufe. Zeitschrift für Pädagogische Psychologie, 20 (1/2), 27-39.

Krapp, A. (1998). Entwicklung und Förderung von Interesse im Unterricht. Psychologie in Erziehung und Unterricht, 44 (3), 185-201.

Kunter, M. \& Baumert, J. (2006a). Who is the expert? Construct and criteria validity of student and teacher ratings of instruction. Learning Environments Research, 9 (3), 231-251.

Kunter, M. \& Baumert, J. (2006b). Linking TIMSS to research on learning and instruction: A re-analysis of the German TIMSS and TIMSS video data. In S. J. Howie \& T. Plomp (Eds.), Contexts of learning mathematics and science. Lessons learned from TIMSS (pp. 335351). London, New York: Routledge.

Lautenschlager, G. J. (1989). A comparison of alternatives to conducting Monte Carlo analyses for determining parallel analysis criteria. Multivariate Behavioral Research, 24, 365-395.

Levelt, W. (1989). Speaking: From intention to articulation. Cambridge: Cambridge University Press.

Lightbown, P. (2000). Classroom SLA research and second language teaching. Anniversary article. Aplied Linguistics, 21 (4), 431-462.

Lüdi, G., Pekarek, S. \& Saudan, V. (2001). Französischlernen in der Deutschschweiz. Chur/Zürich: Rüegger.

Lüdtke, O., Robitzsch, A., Trautwein, U. \& Kunter, M. (2007). Assessing features of the learning environment: How to use student ratings in multilevel modeling. Manuscript submitted for publication.

Lüdtke, O., Trautwein, U., Kunter, M. \& Baumert, J. (2006). Analyse von Lernumwelten. Ansätze zur Bestimmung der Reliabilität und Übereinstimmung von Schülerwahrnehmungen. Zeitschrift für Pädagogische Psychologie, 20 (1/2), 85-96.

Lüdtke, O., Trautwein, U., Schnyder, I. \& Niggli, A. (2007). Simultane Analysen auf Schüler- und Klassenebene: Eine Demonstration der konfirmatorischen Mehrebenen-Faktorenanalyse zur Analyse der Schülerwahrnehmungen am Beispiel der Hausaufgabenvergabe. Zeitschrift für Entwicklungspsychologie und Pädagogische Psychologie, 39, 1-11.

Marsh, H. W., Trautwein, U., Lüdtke, O., Köller, O. \& Baumert, J. (2005). Academic selfconcept, interest, grades and standardized test scores: Reciprocal effect models of causal ordering. Child Development, 76 (2), 397-416.

Meyer, M. A. (2001). Englischunterricht aus allgemeindidaktischer Sicht. In H. E. Tenorth (Hrsg.), Kerncurriculum Oberstufe. Mathematik - Deutsch - Englisch. Expertisen im Auftrag der Ständigen Konferenz der Kultusminister. Weinheim: Beltz.

Neumann, M., Schnyder, I., Trautwein, U., Niggli, A., Lüdtke, O. \& Cathomas, R. (2007). Schulformen als differenzielle Lernmilieus: Institutionelle und kompositionelle Effekte auf die Leistungsentwicklung im Fach Französisch. Zeitschrift für Erziehungswissenschaft, $10(3), 399-420$.

Neumann, M. \& Trautwein, U. (2007). Dornröschen wachgeküsst? Die empirische Schulleistungsforschung und der Fremdsprachenunterricht. Beiträge zur Lehrerbildung, 25 (2), 192-204.

Norris, J. M. \& Ortega, L. (2000). Effectiveness of L2 instruction: A research synthesis and quantitative meta-analysis. Language Learning, 50 (3), 417-528.

Norris, J. M. \& Ortega, L. (2001). Does type of instruction makes a difference? Substantive Findings from a meta-analytic review. Language Learning, 51 (1), 157-213. 
Piepho, H. E. (1976). Kommunikative Didaktik des Englischunterrichts Sekundarstufe I. Theoretische Begründung und Wege zur praktischen Einlösung eines fachdidaktischen Konzeptes. Limburg: Frankonius Verlag.

Raudenbush, S. W. \& Bryk, A. S. (2002). Hierarchical linear models (2nd ed.). Thousand Oaks, CA: Sage.

Raudenbush, S., Bryk, A., Cheong, Y. F., Congdon, R. \& du Toit, M. (2004). HLM 6 hierarchical linear and nonlinear modeling. Lincolnwood: Scientific Software International.

Sammons, P., Elliot, K., Sylva, K., Melhuish, E., Siraj-Blatchford, I. \& Taggart, B. (2004). The impact of pre-school on young children's cognitive attainments at entry to reception. British Educational Research Journal, 30, 691-712.

Saudan, V. (2003). Approche communicative et pédagogie des échanges. Basel: Universität, Romanisches Seminar.

Scherfer, P. (2003). Wortschatzübungen. In K. R. Bausch, H. Christ \& H. J. Krumm (Hrsg.), Handbuch Fremdsprachenunterricht (S. 280-283). Tübingen: Francke.

Schiefele, U., Krapp, A. \& Schreyer, I. (1993). Metaanalyse des Zusammenhangs von Interesse und schulischer Leistung. Zeitschrift für Entwicklungspsychologie und Pädagogische Psychologie, 25 (1), 120-148.

Schiffler, L. (1985). Interaktiver Fremdsprachenunterricht. Stuttgart: Klett.

Schnabel, K. (2001). Psychologie der Lernumwelt. In A. Krapp \& B. Weidenmann (Hrsg.), Pädagogische Psychologie (S. 467-513). Weinheim: Beltz.

Schneider, G. (2007). Fremdsprachenforschung und die Ausbildung von

Fremdsprachenlehrerinnen und -lehrern. Beiträge zur Lehrerbildung, 25 (2), 143-155.

Schnyder, I., Niggli, A., Cathomas, R. \& Trautwein, U. (2006). Unterscheidet sich das Hausaufgabenverhalten von Schülern aus unterschiedlichen Klassen? Befunde einer Mehrebenenanalyse im Fach Französisch. Schweizerische Zeitschrift für Bildungswissenschaften, 28 (2), 295-314.

Schnyder, I., Niggli, A., Cathomas, R., Trautwein, U. \& Lüdtke, O. (2006). Wer lange lernt, lernt noch lange nicht viel mehr: Korrelate der Hausaufgabenzeit im Fach Französich und Effekte auf die Leistungsentwicklung. Psychologie in Erziehung und Unterricht, 53 (2), 107-121.

Stern, O., Eriksson, B., Le Pape Racine, C., Reutener, H. \& Serra, C. (1999). Französisch Deutsch. Zweisprachiges Lernen auf der Sekundarstufe I. Chur/Zürich: Rüegger.

Trautwein, U., Köller, O., Lehmann, R. \& Lüdtke, O. (2007). Schulleistungen von Abiturienten: Regionale, schulformbezogene und soziale Disparitäten. Münster: Waxmann.

Tsai, Y.-M., Kunter, M., Lüdtke, O., Trautwein, U. \& Ryan, R. (in press). What makes lessons interesting? The role of situation and person factors in three school subjects. Journal of Educational Psychology.

Tschirner, E. (2004). Der Wortschatzstand von Studierenden zu Beginn ihres Anglistikstudiums. Fremdsprachen lehren und lernen, 33, 114-127.

Tymms, P. (2004). Effect sizes in multilevel models. In I. Schagen \& K. Elliot (Eds.), But what does it mean? The use of effect sizes in educational research (pp. 55-66). London: National Foundation for Educational Research.

Valette, R. \& Disick, R. S. (1972). Modern language performance objectives and individualization. New York: Harcourt.

Vollmer, H. J., Henrici, G., Finkbeiner, C., Grotjahn, R., Schmid-Schönbein, G. \& Zydatiss, W. (2001). Lernen und Lehren von Fremdsprachen: Kognition, Affektion, Interaktion. Ein Forschungsüberblick. Zeitschrift für Fremdsprachenforschung, 12 (2), 1-145.

Ziberi-Luginbühl, J. (1999). Zweitsprachunterricht im obligatorischen Schulsystem. Aarau: NFP 33, SKBF.

Zydatiss, W. (2000). Bilingualer Unterricht in der Grundschule. Entwurf eines Spracherwerbskonzepts für zweisprachige Immersionsprogramme. Ismaning: Hueber. 
Schlagworte: Fremdsprachenlernen, Fachdidaktik, Unterrichtsqualität, Schulleistung, Fachinteresse, Mehrebenenanalyse

\section{Les interventions didactiques dans l'enseignement du français du point de vue des élèves: Leur relation avec les performances scolaires et l'intérêt}

Résumé

La recherche récente sur la qualité de l'enseignement des langues étrangères a démontré la nécessité de compléter le modèle des dimensions interdisciplinaires de la qualité de l'enseignement avec des dimensions spécifiques aux disciplines. L'étude présentée ici examine des présupposés théoriques quant aux pratiques didactiques qui sont déterminantes dans les performances et l'intérêt que développent les élèves pour l'enseignement des langues étrangères. Elle est réalisée avec un échantillon de 1655 élèves de huitième année dans trois cantons suisses: Fribourg, Lucerne et Valais. L'analyse factorielle a permis d'identifier cinq facteurs pour l'enseignement du français comme langue étrangère: utilisation de la langue française durant l'enseignement, intensité de l'exercice (de la répétition) et de l'application du savoir, utilisation de matériels issus de la vie quotidienne, apprentissage du vocabulaire et communication avec des personnes de langue française. Des analyses multiniveaux ont démontré que l'utilisation de la langue française, ainsi que l'exercice et l'application intensifs du savoir sont positivement associés aux performances et à l'intérêt que les élèves développent pour la discipline au cours d'une année. L'apprentissage du vocabulaire demeure par contre sans influence. Contrairement aux attentes, une relation négative est constatée entre la communication avec des personnes de langue française et le développement de l'intérêt pour la langue concernée.

Mots clés: apprentissage des langues étrangères, performance scolaire, intérêt pour une discipline, analyse multiniveaux

\section{Misure didattiche specifiche adottate nell'insegnamento del francese considerate dalla prospettiva degli studenti. Relazioni tra rendimento e motivazione.}

\section{Riassunto}

La recente ricerca sulla qualità dell'insegnamento delle lingue straniere segnala la necessità di completare la dimensione interdisciplinare dell'insegnamento con modelli descrittivi specifici ai vari settori. Nel contributo sono state esaminate delle ipotesi teoriche sulle determinanti di rendimento e di interesse nell'insegnamento delle lingue straniere. Per analizzare tali fattori si è ricorso a un campione di 1655 studenti dell'ottavo anno di tre cantoni svizzeri (Friburgo, Lu- 
cerna e Vallese). Per l'insegnamento del francese come lingua straniera sono stati messi in luce cinque fattori: utilizzo del francese nell'insegnamento, intensità delle esercitazioni e delle applicazioni, utilizzo di materiale proveniente dalla vita quotidiana, apprendimento del lessico e comunicazione con persone di lingua francese. Analizzando in seguito i risultati su diversi livelli, è stato possibile dimostrare che l'utilizzo del francese associato all'applicazione e all'esercitazione intensa porta, sull'arco di un anno, a uno sviluppo positivo del rendimento e dell'interesse per la lingua. L'apprendimento dei vocaboli è rimasto invariato. Contrariamente alle aspettative, è stato invece registrato un rapporto negativo tra la comunicazione con le persone di lingua francese e lo sviluppo di interesse per la lingua.

Parole chiave: apprendimento di lingue straniere, rendimento scolastico, interesse per la materia, analisi multilivello

\section{Students' perceptions of domain-specific teaching practices in French as a second language: Their relationships with school performances and interest}

\footnotetext{
Abstract

Research on the quality of second language teaching has recently highlighted the need to consider not only cross-curricular but also domain-specific dimensions of instructional quality. In this study, theoretical assumptions about domain-specific determinants of interest and performances in second language acquisition are tested, using data from a sample of 1655 eighth graders in three Swiss cantons: Fribourg, Lucerne, and Valais. Factor analysis yielded five factors related to teaching dimensions of French as an additional language: use of French in lessons, intensity of language use and practice, use of authentic material, vocabulary learning, and communication with native speakers. Subsequent multilevel analyses showed that using French in lessons, as well as intensive language use and practice are significantly associated with the students performances and the interest the develop over a 1-year period. Vocabulary learning has no effect. Contrary to expectations, there is a negative relationship between communicating with French native speakers and the development of interest for learning French as a second language.
}

Key words: second language learning, student achievement, domain-specific interest, multilevel analysis 


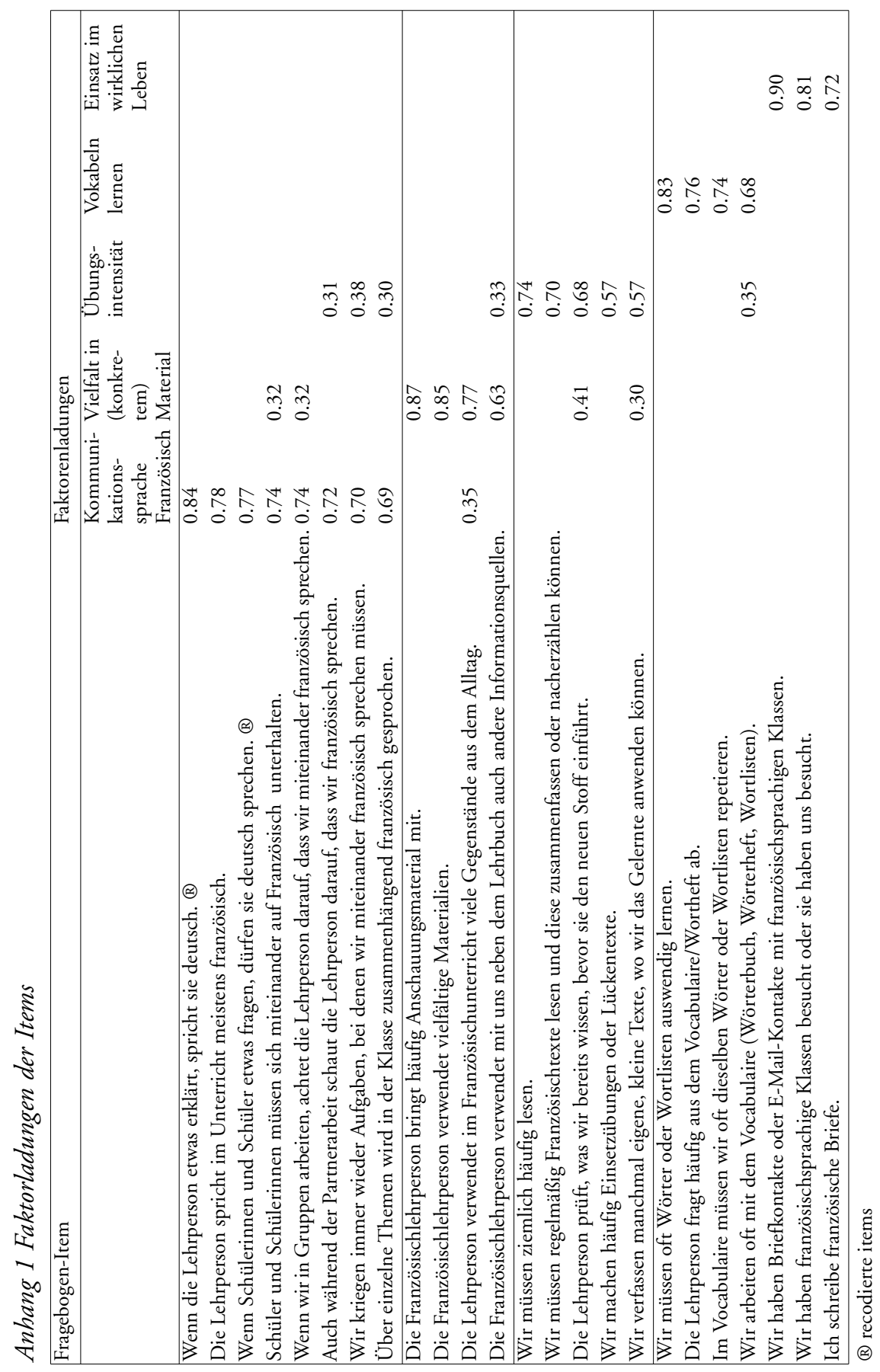


Eliminierte Items:

Wir Schülerinnen und Schüler müssen oft zusammen Französischaufgaben lösen.

Die Lehrperson bietet den Schülerinnen und Schülern an, hin und wieder ein eigenes Thema auf Französisch zu bearbeiten.

Grammatik trainieren wir hauptsächlich mit einfachen Sätzen, an denen wir die Regel sofort erkennen.

Bei dieser Lehrperson arbeiten wir meistens mit dem Lehrbuch.

Wir behandeln auch Texte, mit denen wir die Menschen und das Leben aus dem französischen Sprachgebiet besser kennen lernen.

Wir machen kleine Rollenspiele in französischer Sprache (z.B. beim Essen, am Bahnschalter, beim Telefonieren) 